\title{
GLOBAL EXISTENCE OF SOLUTIONS TO MULTIPLE SPEED SYSTEMS OF QUASILINEAR WAVE EQUATIONS IN EXTERIOR DOMAINS
}

\author{
JASON METCALFE, MAKOTO NAKAMURA, AND CHRISTOPHER D. SOGGE
}

1. Introduction. The goal of this paper is to prove global existence of solutions to quadratic quasilinear Dirichlet-wave equations exterior to a class of compact obstacles. As in Metcalfe-Sogge 22, the main condition that we require for our class of obstacles is exponential local energy decay (with a possible loss of regularity). Our result improves upon the earlier one of Metcalfe-Sogge 22] by allowing a more general null condition which only puts restrictions on the self-interaction of each wave family. The nonrelativistic system that we study serves as a simplified model for the equations of elasticity. In Minkowski space, such equations were studied and shown to have global solutions by Sideris-Tu 29], Agemi-Yokoyama [1], and Kubota-Yokoyama [18.

The null condition we use here is the natural one for systems of quasilinear wave equations with multiple speeds. Following an observation of John and Shatah, this null condition is equivalent to the requirement that no plane wave solution of the system is genuinely nonlinear (see John [1], p. 23 for the single-speed case and Agemi and Yokoyama [1] for the multi-speed case). In order to allow the more general null condition, instead of just exploring a coupling between a low order dispersive estimate and higher order energy estimates as in Metcalfe-Sogge [22], we must first develop a low order energy estimate and couple this with a low order pointwise estimate on the gradient and higher order energy estimates. Thus, our approach is a blend of the ones using pointwise estimates based on fundamental solutions (see e.g., 12, [14, 18, [22, [32, 33]) and ones using more refined $L^{2}$ energy estimates for lower order terms (see, e.g. [5], 27, 28, [29]). As in the approach first developed in [13] weighted space-time $L^{2}$ estimates for lower order terms will also play a key role in our arguments.

We will be using an exterior domain analog of Klainerman's commuting vector fields method [16]. Here, we have to restrict to the collection of vector fields that are "admissible" for boundary value problems, $\{Z, L\}$, where $Z$ denotes the generators of the spatial rotations and space-time translations

$$
Z=\left\{\partial_{i}, x_{j} \partial_{k}-x_{k} \partial_{j}, 0 \leq i \leq 3,1 \leq j<k \leq 3\right\}
$$

and $L$ is the scaling vector field

$$
L=t \partial_{t}+r \partial_{r} .
$$

Here and in what follows, $r=|x|$, and we will write

$$
\Omega_{i j}=x_{i} \partial_{j}-x_{j} \partial_{i}, \quad 1 \leq i<j \leq 3 .
$$

The first and third authors were supported in part by the NSF. 
The generators of the hyperbolic rotations, $x_{i} \partial_{t}+t \partial_{i}$, have an associated speed and the coefficients are unbounded on the boundary of the obstacle, and thus, they do not seem appropriate for the problem in question.

In Minkowski space, since $\left[\left(\partial_{t}^{2}-\Delta\right), Z\right]=0$ and $\left[\left(\partial_{t}^{2}-\Delta\right), L\right]=2\left(\partial_{t}^{2}-\Delta\right)$, we see that $Z$ and $L$ preserve the equation $\left(\partial_{t}^{2}-\Delta\right) u=0$. This is no longer the case in the exterior domain since the Dirichlet boundary condition is not preserved. For the vector fields $Z$, since their coefficients remain small in a neighborhood of our compact obstacle, this is fairly easy to get around. On the other hand, since the coefficients of $L$ are large near the obstacle as $t \rightarrow \infty$, we must stick to estimates that require relatively few of the scaling vector field.

As in 13, 14, we will use weighted $L_{t}^{2} L_{x}^{2}$ estimates where the weight is just a negative power of $\langle x\rangle=\langle r\rangle=\sqrt{1+r^{2}}$. These estimates are useful for handling the lower order terms that arise in the study of such boundary value problems. They permit us to use pointwise estimates for linear, inhomogeneous wave equations with $O\left(\langle x\rangle^{-1}\right)$ decay rather than the more standard $O\left(t^{-1}\right)$ decay which is more difficult to prove in the obstacle setting. Additionally, such estimates allow us, as in [22, to handle the boundary terms that arise in the energy estimates if the obstacle is no longer assumed to be star-shaped. Here we exploit the fact that we are studying equations with quadratic nonlinearities.

Additionally, we will be developing exterior domain analogs of a class of weighted Sobolev estimates. The weights here will involve powers of $r$ and $\langle t-r\rangle$. Specifically, we will be looking at estimates of Klainerman-Sideris [17 and Hidano-Yokoyama 6]. We would additionally like to mention the works of Hidano [5], Kubota-Yokoyama [1], Sideris [27, 28], and Sideris-Tu [29] where similar estimates were used for the boundaryless case.

At this point, we wish to describe our assumptions on our obstacles $\mathcal{K} \subset \mathbb{R}^{3}$. We shall assume that $\mathcal{K}$ is smooth and compact, but not necessarily connected. By scaling, without loss of generality, we may assume

$$
\mathcal{K} \subset\left\{x \in \mathbb{R}^{3}:|x|<1\right\}
$$

throughout. The only additional assumption states that there is exponential local energy decay with a possible loss of regularity. That is, if $u$ is a solution to $\square u=0$ with Cauchy data $u(0, x), \partial_{t} u(0, x)$ supported in $|x| \leq 4$, then there must be constants $c, C>0$ so that

$$
\left(\int_{\left\{x \in \mathbb{R}^{3} \backslash \mathcal{K}:|x|<4\right\}}\left|u^{\prime}(t, x)\right|^{2} d x\right)^{1 / 2} \leq C e^{-c t} \sum_{|\alpha| \leq 1}\left\|\partial_{x}^{\alpha} u^{\prime}(0, \cdot)\right\|_{2} .
$$

Here, and throughout, we are taking $\partial=\nabla_{t, x}$ to be the space-time gradient.

We note that we do not require exponential decay; in fact, $O\left((1+t)^{-1-\delta}\right)$ may be sufficient with a tighter argument. For simplicity, we will assume (1.4). Currently, the authors are not aware of any 3-dimensional example that involves polynomial decay, but does not have exponential decay.

Notice that if the obstacle is assumed to be nontrapping, then a stronger version of (1.4) holds where $\alpha=0$ on the right side (see, e.g., Morawetz-Ralston-Strauss [24]). If there are trapped rays, it was shown by Ralston [25] that (1.4) could not hold without a 
loss of regularity $\ell>0$ in the right side. We will assume throughout that $\ell=1$. This can be done without loss of generality since if $\ell>1$, interpolation with the standard energy inequality will yield (1.4) (with a different constant $c$ ). In fact, we could take $\ell=\delta$ for any $\delta>0$.

Ikawa [9], 10] showed that there was such exponential decay of local energy for certain finite unions of smooth, convex obstacles with a loss $\ell=7$. In particular, using Ikawa's result, we have (1.4) for two disjoint convex obstacles or any number of sufficiently separated balls.

For such smooth, compact obstacles $\mathcal{K} \subset \mathbb{R}^{3}$ satisfying (1.4), we shall consider quadratic, quasilinear systems of the form

$$
\left\{\begin{array}{l}
\square u=Q\left(d u, d^{2} u\right), \quad(t, x) \in \mathbb{R}_{+} \times \mathbb{R}^{3} \backslash \mathcal{K} \\
\left.u(t, \cdot)\right|_{\partial \mathcal{K}}=0 \\
u(0, \cdot)=f, \quad \partial_{t} u(0, \cdot)=g
\end{array}\right.
$$

Here

$$
\square=\left(\square_{c_{1}}, \square_{c_{2}}, \ldots, \square_{c_{D}}\right)
$$

is a vector-valued multiple speed d'Alembertian with

$$
\square_{c_{I}}=\partial_{t}^{2}-c_{I}^{2} \Delta \text {. }
$$

We will assume that the wave speeds $c_{I}$ are positive and distinct. This situation is referred to as the nonrelativistic case. Straightforward modifications of the argument give the more general case where the various components are allowed to have the same speed. Also, $\Delta=\partial_{1}^{2}+\partial_{2}^{2}+\partial_{3}^{2}$ is the standard Laplacian. Additionally, when convenient, we will allow $x_{0}=t$ and $\partial_{0}=\partial_{t}$.

We shall assume that $Q\left(d u, d^{2} u\right)$ is of the form

$$
Q^{I}\left(d u, d^{2} u\right)=B^{I}(d u)+\sum_{\substack{0 \leq j, k, l \leq 3 \\ 1 \leq J, K \leq D}} B_{K, l}^{I J, j k} \partial_{l} u^{K} \partial_{j} \partial_{k} u^{J}, \quad 1 \leq I \leq D
$$

where $B^{I}(d u)$ is a quadratic form in the gradient of $u$ and $B_{K, l}^{I J, j k}$ are real constants satisfying the symmetry conditions

$$
B_{K, l}^{I J, j k}=B_{K, l}^{J I, j k}=B_{K, l}^{I J, k j} .
$$

To obtain global existence, we shall also require that the equations satisfy the following null condition which only involves the self-interactions of each wave family. That is, we require that

$$
\sum_{0 \leq j, k, l \leq 3} B_{J, l}^{J J, j k} \xi_{j} \xi_{k} \xi_{l}=0 \quad \text { whenever } \quad \frac{\xi_{0}^{2}}{c_{J}^{2}}-\xi_{1}^{2}-\xi_{2}^{2}-\xi_{3}^{2}=0, \quad J=1, \ldots, D .
$$

To describe the null condition for the lower order terms, we expand

$$
B^{I}(d u)=\sum_{\substack{1 \leq J, K \leq D \\ 0 \leq j, k \leq 3}} A_{J K}^{I, j k} \partial_{j} u^{J} \partial_{k} u^{K}
$$


We then require that each component satisfies the similar null condition

$$
\sum_{0 \leq j, k \leq 3} A_{J J}^{J, j k} \xi_{j} \xi_{k}=0 \quad \text { whenever } \quad \frac{\xi_{0}^{2}}{c_{J}^{2}}-\xi_{1}^{2}-\xi_{2}^{2}-\xi_{3}^{2}=0, \quad J=1, \ldots, D .
$$

In order to solve (1.5) we must also assume that the data satisfies the relevant compatibility conditions. Since these are well-known (see, e.g., 12 ), we shall only describe them briefly. To do so we first let $J_{k} u=\left\{\partial_{x}^{\alpha} u: 0 \leq|\alpha| \leq k\right\}$ denote the collection of all spatial derivatives of $u$ of order up to $k$. Then if $m$ is fixed and if $u$ is a formal $H^{m}$ solution of (1.5), we can write $\partial_{t}^{k} u(0, \cdot)=\psi_{k}\left(J_{k} f, J_{k-1} g\right), 0 \leq k \leq m$, for certain compatibility functions $\psi_{k}$ which depend on the nonlinear term $Q$ as well as $J_{k} f$ and $J_{k-1} g$. Having done this, the compatibility condition for 1.5 with $(f, g) \in H^{m} \times H^{m-1}$ is just the requirement that the $\psi_{k}$ vanish on $\partial \mathcal{K}$ when $0 \leq k \leq m-1$. Additionally, we shall say that $(f, g) \in C^{\infty}$ satisfy the compatibility conditions to infinite order if this condition holds for all $m$.

We can now state our main result:

Theorem 1.1. Let $\mathcal{K}$ be a fixed compact obstacle with smooth boundary that satisfies (1.4). Assume that $Q\left(d u, d^{2} u\right)$ and $\square$ are as above and that $(f, g) \in C^{\infty}\left(\mathbb{R}^{3} \backslash \mathcal{K}\right)$ satisfy the compatibility conditions to infinite order. Then there is a constant $\varepsilon_{0}>0$, and an integer $N>0$ so that for all $\varepsilon<\varepsilon_{0}$, if

$$
\sum_{|\alpha| \leq N}\left\|<x>^{|\alpha|} \partial_{x}^{\alpha} f\right\|_{2}+\sum_{|\alpha| \leq N-1}\left\|<x>^{1+|\alpha|} \partial_{x}^{\alpha} g\right\|_{2} \leq \varepsilon
$$

then (1.5) has a unique solution $u \in C^{\infty}\left([0, \infty) \times \mathbb{R}^{3} \backslash \mathcal{K}\right)$.

This paper is organized as follows. In the next section, we will recall some energy estimates from [22]. In $\S 3$, we will gather the pointwise estimates that we will require. In $\S 4$, we will collect some Sobolev-type estimates. Included are some bounds on the null forms which are exterior domain analogs of those from [29] and 32. Finally, in $\S 5$, we will use these estimates to prove the global existence theorem via a continuity argument.

2. $L^{2}$ Estimates. In this section, we will recall some estimates of 22 related to the energy inequality. Unless stated otherwise, the proofs of the following results can be found in $\left[22\right.$. Specifically, we will be concerned with solutions $u \in C^{\infty}\left(\mathbb{R}_{+} \times \mathbb{R}^{3} \backslash \mathcal{K}\right)$ of the Dirichlet-wave equation

$$
\left\{\begin{array}{l}
\square_{\gamma} u=F \\
\left.u\right|_{\partial \mathcal{K}}=0 \\
\left.u\right|_{t=0}=f,\left.\quad \partial_{t} u\right|_{t=0}=g
\end{array}\right.
$$

where

$$
\left(\square_{\gamma} u\right)^{I}=\left(\partial_{t}^{2}-c_{I}^{2} \Delta\right) u^{I}+\sum_{J=1}^{D} \sum_{j, k=0}^{3} \gamma^{I J, j k}(t, x) \partial_{j} \partial_{k} u^{J}, \quad 1 \leq I \leq D .
$$

We shall assume that the $\gamma^{I J, j k}$ satisfy the symmetry conditions

$$
\gamma^{I J, j k}=\gamma^{J I, j k}=\gamma^{I J, k j}
$$


as well as the size condition

$$
\sum_{I, J=1}^{D} \sum_{j, k=0}^{3}\left\|\gamma^{I J, j k}(t, x)\right\|_{\infty} \leq \delta
$$

for $\delta$ sufficiently small (depending on the wave speeds). The energy estimate will involve bounds for the gradient of the perturbation terms

$$
\left\|\gamma^{\prime}(t, \cdot)\right\|_{\infty}=\sum_{I, J=1}^{D} \sum_{j, k, l=0}^{3}\left\|\partial_{l} \gamma^{I J, j k}(t, \cdot)\right\|_{\infty}
$$

and the energy form associated with $\square_{\gamma}, e_{0}(u)=\sum_{I=1}^{D} e_{0}^{I}(u)$, where

$$
\begin{aligned}
& e_{0}^{I}(u)=\left(\partial_{0} u^{I}\right)^{2}+\sum_{k=1}^{3} c_{I}^{2}\left(\partial_{k} u^{I}\right)^{2} \\
& \quad+2 \sum_{J=1}^{D} \sum_{k=0}^{3} \gamma^{I J, 0 k} \partial_{0} u^{I} \partial_{k} u^{J}-\sum_{J=1}^{D} \sum_{j, k=0}^{3} \gamma^{I J, j k} \partial_{j} u^{I} \partial_{k} u^{J} .
\end{aligned}
$$

The most basic estimate will lead to a bound for

$$
E_{M}(t)=E_{M}(u)(t)=\int \sum_{j=0}^{M} e_{0}\left(\partial_{t}^{j} u\right)(t, x) d x
$$

Lemma 2.1. Fix $M=0,1,2, \ldots$, and assume that the perturbation terms $\gamma^{I J, j k}$ are as above. Suppose also that $u \in C^{\infty}$ solves (2.1) and for every $t, u(t, x)=0$ for large $x$. Then there is an absolute constant $C$ so that

$$
\partial_{t} E_{M}^{1 / 2}(t) \leq C \sum_{j=0}^{M}\left\|\square_{\gamma} \partial_{t}^{j} u(t, \cdot)\right\|_{2}+C\left\|\gamma^{\prime}(t, \cdot)\right\|_{\infty} E_{M}^{1 / 2}(t) .
$$

Before stating the next result, let us introduce some notation. If $P=P\left(t, x, D_{t}, D_{x}\right)$ is a differential operator, we shall let

$$
\left[P, \gamma^{k l} \partial_{k} \partial_{l}\right] u=\sum_{1 \leq I, J \leq D} \sum_{0 \leq k, l \leq 3}\left|\left[P, \gamma^{I J, k l} \partial_{k} \partial_{l}\right] u^{J}\right|
$$

In order to generalize the above energy estimate to include the more general vector fields $L, Z$, we will need to use a variant of the scaling vector field $L$. We fix a bump function $\eta \in C^{\infty}\left(\mathbb{R}^{3}\right)$ with $\eta(x)=0$ for $x \in \mathcal{K}$ and $\eta(x)=1$ for $|x|>1$. Then, set $\tilde{L}=\eta(x) r \partial_{r}+t \partial_{t}$. Using this variant of the scaling vector field and an elliptic regularity argument, one can establish

Proposition 2.2. Suppose that the constant in (2.3) is small. Suppose further that

$$
\left\|\gamma^{\prime}(t, \cdot)\right\|_{\infty} \leq \delta /(1+t)
$$


and

$$
\begin{aligned}
\sum_{\substack{j+\mu \leq N_{0}+\nu_{0} \\
\mu \leq \nu_{0}}}\left(\left\|\tilde{L}^{\mu} \partial_{t}^{j} \square_{\gamma} u(t, \cdot)\right\|_{2}+\right. & \left.\left\|\left[\tilde{L}^{\mu} \partial_{t}^{j}, \gamma^{k l} \partial_{k} \partial_{l}\right] u(t, \cdot)\right\|_{2}\right) \\
& \leq \frac{\delta}{1+t} \sum_{\substack{j+\mu \leq N_{0}+\nu_{0} \\
\mu \leq \nu_{0}}}\left\|\tilde{L}^{\mu} \partial_{t}^{j} u^{\prime}(t, \cdot)\right\|_{2}+H_{\nu_{0}, N_{0}}(t),
\end{aligned}
$$

where $N_{0}$ and $\nu_{0}$ are fixed. Then

$$
\sum_{\substack{|\alpha|+\mu \leq N_{0}+\nu_{0} \\ \mu \leq \nu_{0}}}\left\|L^{\mu} \partial^{\alpha} u^{\prime}(t, \cdot)\right\|_{2}
$$

$$
\begin{gathered}
\leq C \sum_{\substack{|\alpha|+\mu \leq N_{0}+\nu_{0}-1 \\
\mu \leq \nu_{0}}}\left\|L^{\mu} \partial^{\alpha} \square u(t, \cdot)\right\|_{2}+C(1+t)^{A \delta} \sum_{\substack{\mu+j \leq N_{0}+\nu_{0} \\
\mu \leq \nu_{0}}}\left(\int e_{0}\left(\tilde{L}^{\mu} \partial_{t}^{j} u\right)(0, x) d x\right)^{1 / 2} \\
+C(1+t)^{A \delta}\left(\int_{0}^{t} \sum_{\substack{|\alpha|+\mu \leq N_{0}+\nu_{0}-1 \\
\mu \leq \nu_{0}-1}}\left\|L^{\mu} \partial^{\alpha} \square u(s, \cdot)\right\|_{2} d s+\int_{0}^{t} H_{\nu_{0}, N_{0}}(s) d s\right) \\
+C(1+t)^{A \delta} \int_{0}^{t} \sum_{\substack{|\alpha|+\mu \leq N_{0}+\nu_{0} \\
\mu \leq \nu_{0}-1}}\left\|L^{\mu} \partial^{\alpha} u^{\prime}(s, \cdot)\right\|_{L^{2}(|x|<1)} d s
\end{gathered}
$$

where the constants $C$ and $A$ are absolute constants.

In practice $H_{\nu_{0}, N_{0}}(t)$ will involve weighted $L_{x}^{2}$ norms of $\left|L^{\mu} \partial^{\alpha} u^{\prime}\right|^{2}$ with $\mu+|\alpha|$ much smaller than $N_{0}+\nu_{0}$, and so the integral involving $H_{\nu_{0}, N_{0}}$ can be dealt with using an inductive argument and weighted $L_{t}^{2} L_{x}^{2}$ estimates that will be presented at the end of this section.

In proving our existence results for (1.5), the key step will be to obtain a priori $L^{2}$ estimates involving $L^{\mu} Z^{\alpha} u^{\prime}$. Begin by setting

$$
Y_{N_{0}, \nu_{0}}(t)=\int \sum_{\substack{|\alpha|+\mu \leq N_{0}+\nu_{0} \\ \mu \leq \nu_{0}}} e_{0}\left(L^{\mu} Z^{\alpha} u\right)(t, x) d x .
$$

We, then, have the following proposition which shows how the $L^{\mu} Z^{\alpha} u^{\prime}$ estimates can be obtained from the ones involving $L^{\mu} \partial^{\alpha} u^{\prime}$.

Proposition 2.3. Suppose that the constant $\delta$ in (2.3) is small and that (2.6) holds. Then,

$$
\begin{aligned}
\partial_{t} Y_{N_{0}, \nu_{0}} \leq C Y_{N_{0}, \nu_{0}}^{1 / 2} \sum_{\substack{|\alpha|+\mu \leq N_{0}+\nu_{0} \\
\mu \leq \nu_{0}}}\left\|\square_{\gamma} L^{\mu} Z^{\alpha} u(t, \cdot)\right\|_{2}+C\left\|\gamma^{\prime}(t, \cdot)\right\|_{\infty} Y_{N_{0}, \nu_{0}} \\
+C \sum_{\substack{|\alpha|+\mu \leq N_{0}+\nu_{0}+1 \\
\mu \leq \nu_{0}}}\left\|L^{\mu} \partial^{\alpha} u^{\prime}(s, \cdot)\right\|_{L^{2}(|x|<1)}^{2} .
\end{aligned}
$$


As in 13 and 14 we shall also require some weighted $L_{t}^{2} L_{x}^{2}$ estimates. They will be used, for example, to control the local $L^{2}$ norms such as the last term in (2.10). For convenience, for the remainder of this section, allow $\square=\partial_{t}^{2}-\Delta$ to denote the unit speed d'Alembertian. The transition from the following estimates to those involving (1.6) is straightforward. Also, allow

$$
S_{T}=\left\{[0, T] \times \mathbb{R}^{3} \backslash \mathcal{K}\right\}
$$

to denote the time strip of height $T$ in $\mathbb{R}_{+} \times \mathbb{R}^{3} \backslash \mathcal{K}$.

We, then, have the following proposition.

Proposition 2.4. Fix $N_{0}$ and $\nu_{0}$. Suppose that $\mathcal{K}$ satisfies the local exponential energy decay (1.4). Suppose further that $u \in C^{\infty}$ solves (2.1) and $u(t, x)=0$ for $t<0$. Then there is a constant $C=C_{N_{0}, \nu_{0}, \mathcal{K}}$ so that if $u$ vanishes for large $x$ at every fixed $t$

$$
\begin{aligned}
& (\log (2+T))^{-1 / 2} \sum_{\substack{|\alpha|+\mu \leq N_{0}+\nu_{0} \\
\mu \leq \nu_{0}}}\left\|<x>^{-1 / 2} L^{\mu} \partial^{\alpha} u^{\prime}\right\|_{L^{2}\left(S_{T}\right)} \\
& \leq C \int_{0}^{T} \sum_{\substack{|\alpha|+\mu \leq N_{0}+\nu_{0}+1 \\
\mu \leq \nu_{0}}}\left\|\square L^{\mu} \partial^{\alpha} u(s, \cdot)\right\|_{2} d s+C \sum_{\substack{|\alpha|+\mu \leq N_{0}+\nu_{0} \\
\mu \leq \nu_{0}}}\left\|\square L^{\mu} \partial^{\alpha} u\right\|_{L^{2}\left(S_{T}\right)}
\end{aligned}
$$

and

$$
\begin{aligned}
& (\log (2+T))^{-1 / 2} \sum_{\substack{|\alpha|+\mu \leq N_{0}+\nu_{0} \\
\mu \leq \nu_{0}}}\left\|<x>^{-1 / 2} L^{\mu} Z^{\alpha} u^{\prime}\right\|_{L^{2}\left(S_{T}\right)} \\
& \leq C \int_{0}^{T} \sum_{\substack{|\alpha|+\mu \leq N_{0}+\nu_{0}+1 \\
\mu \leq \nu_{0}}}\left\|\square L^{\mu} Z^{\alpha} u(s, \cdot)\right\|_{2} d s+C \sum_{\substack{|\alpha|+\mu \leq N_{0}+\nu_{0} \\
\mu \leq \nu_{0}}}\left\|\square L^{\mu} Z^{\alpha} u\right\|_{L^{2}\left(S_{T}\right)} .
\end{aligned}
$$

To be able to handle the last term in (2.8), we shall need the following.

Lemma 2.5. Suppose that (1.4) holds, and suppose that $u \in C^{\infty}$ solves (2.1) and satisfies $u(t, x)=0$ for $t<0$. Then, for fixed $N_{0}$ and $\nu_{0}$ and $t>2$,

$$
\begin{aligned}
& \sum_{\substack{|\alpha|+\mu \leq N_{0}+\nu_{0} \\
\mu \leq \nu_{0}}} \int_{0}^{t}\left\|L^{\mu} \partial^{\alpha} u^{\prime}(s, \cdot)\right\|_{L^{2}(|x|<2)} d s \\
& \quad \leq C \sum_{\substack{|\alpha|+\mu \leq N_{0}+\nu_{0}+1 \\
\mu \leq \nu_{0}}} \int_{0}^{t}\left(\int_{0}^{s}\left\|L^{\mu} \partial^{\alpha} \square u(\tau, \cdot)\right\|_{L^{2}(|| x|-(s-\tau)|<10)} d \tau\right) d s .
\end{aligned}
$$

3. Pointwise Estimates. Here we will estimate solutions of the scalar inhomogeneous wave equation

$$
\left\{\begin{array}{l}
\left(\partial_{t}^{2}-\Delta\right) w(t, x)=F(t, x), \quad(t, x) \in \mathbb{R}_{+} \times \mathbb{R}^{3} \backslash \mathcal{K} \\
\left.w(t, \cdot)\right|_{\partial \mathcal{K}}=0 \\
w(t, x)=0, \quad t \leq 0
\end{array}\right.
$$

If we assume, as before, that $\mathcal{K} \subset\left\{x \in \mathbb{R}^{3}:|x|<1\right\}$ and that $\mathcal{K}$ satisfies (1.4), then we have the following result whose proof can be found in Metcalfe-Sogge [22]. 
Theorem 3.1. Let $w$ be a solution to (3.1), and suppose that the local energy decay bounds (1.4) hold for $\mathcal{K}$. Then,

$$
\begin{aligned}
& (1+t+|x|)\left|L^{\nu} Z^{\alpha} w(t, x)\right| \leq C \int_{0}^{t} \int_{\mathbb{R}^{3} \backslash \mathcal{K}} \sum_{\substack{|\beta|+\mu \leq|\alpha|+\nu+7 \\
\mu \leq \nu+1}}\left|L^{\mu} Z^{\beta} F(s, y)\right| \frac{d y d s}{|y|} \\
& +C \int_{0}^{t} \sum_{\substack{|\beta|+\mu \leq|\alpha|+\nu+4 \\
\mu \leq \nu+1}}\left\|L^{\mu} \partial^{\beta} F(s, \cdot)\right\|_{L^{2}(|y|<2)} d s .
\end{aligned}
$$

Here and throughout $\{|y|<2\}$ is understood to mean $\left\{y \in \mathbb{R}^{3} \backslash \mathcal{K}:|y|<2\right\}$.

Additionally, we can prove the following improved pointwise bound for the gradient of the solution $w$. In this modified result, we are able to bring the gradient inside the main term (the first term) on the right side.

Theorem 3.2. Let $w$ be a solution to (3.1), and suppose that the local energy decay bounds (1.4) hold for $\mathcal{K}$. Suppose further that $F(t, x)=0$ when $|x|>10 t$. Then, if $|x|<t / 10$ and $t>1$,

$$
\begin{aligned}
(1+t+|x|)\left|L^{\nu} Z^{\alpha} w^{\prime}(t, x)\right| \leq C & \sum_{\substack{\mu+|\beta| \leq \nu+|\alpha|+3 \\
\mu \leq \nu+1}} \int_{t / 100}^{t} \int_{\mathbb{R}^{3} \backslash \mathcal{K}}\left|L^{\mu} Z^{\beta} F^{\prime}(s, y)\right| \frac{d y d s}{|y|} \\
& +C \sup _{0 \leq s \leq t}(1+s) \sum_{\substack{|\beta|+\mu \leq|\alpha|+\nu+4 \\
\mu \leq \nu}}\left\|L^{\mu} Z^{\beta} F(s, \cdot)\right\|_{\infty} \\
& +C \sup _{0 \leq s \leq t}(1+s) \sum_{|\beta|+\mu \leq|\alpha|+\nu+7} \int_{0}^{s} \int_{|| y|-(s-\tau)| \leq 10}\left|L^{\mu} Z^{\beta} F(\tau, y)\right| \frac{d y d \tau}{|y|} \\
& +C \sup _{0 \leq s \leq t} \sum_{\substack{|\beta|+\mu \\
|>| \alpha \mid+\nu) / 2}} \int_{s \leq \nu+100}^{s} \int_{|y| \geq(1+\tau) / 10}\left|L^{\mu} Z^{\beta} F(\tau, y)\right| \frac{d y d \tau}{|y|} .
\end{aligned}
$$

The remainder of this section will be dedicated to the proof of (3.3).

The Minkowski space estimate we shall use says that if $w_{0}$ is a solution to the inhomogeneous wave equation

$$
\left\{\begin{array}{l}
\left(\partial_{t}^{2}-\Delta\right) w_{0}(t, x)=G(t, x), \quad(t, x) \in \mathbb{R}_{+} \times \mathbb{R}^{3} \\
w_{0}(0, x)=\partial_{t} w_{0}(0, x)=0
\end{array}\right.
$$

and if $G(s, y)=0$ when $|y|>10 s$, then

$$
(1+t)\left|L^{\nu} Z^{\alpha} w_{0}(t, x)\right| \leq C \int_{t / 100}^{t} \int_{\mathbb{R}^{3}} \sum_{\substack{|\beta|+\mu \leq|\alpha|+\nu+3 \\ \mu \leq \nu+1}}\left|L^{\mu} Z^{\beta} G(s, y)\right| \frac{d y d s}{|y|}, \quad|x|<t / 10 .
$$

By using sharp Huygens principle, this follows from inequality (2.3) in [14] and the fact that $\left[\partial_{t}^{2}-\Delta, Z\right]=0$ and $\left[\partial_{t}^{2}-\Delta, L\right]=2\left(\partial_{t}^{2}-\Delta\right)$. 
Recall that we are assuming that $\mathcal{K} \subset\left\{x \in \mathbb{R}^{3}:|x|<1\right\}$. With this in mind, the first step is to see that (3.5) yields for $|x|<t / 10$

$$
\begin{gathered}
(1+t)\left|L^{\nu} Z^{\alpha} w^{\prime}(t, x)\right| \leq C \int_{t / 100}^{t} \int_{\mathbb{R}^{3} \backslash \mathcal{K}} \sum_{\substack{|\beta|+\mu \leq|\alpha|+\nu+3 \\
\mu \leq \nu+1}}\left|L^{\mu} Z^{\beta} F^{\prime}(s, y)\right| \frac{d y d s}{|y|} \\
\quad+C \sup _{|y| \leq 2,0 \leq s \leq t}(1+s) \sum_{\substack{|\beta|+\mu \leq|\alpha|+\nu+1 \\
\mu \leq \nu}}\left|L^{\mu} \partial^{\beta} w^{\prime}(s, y)\right| .
\end{gathered}
$$

The proof is exactly like that of Lemma 4.2 in [14].

As a result of (3.6), we would be done if we could show that

$$
\sup _{0 \leq s \leq t}(1+s) \sum_{\substack{|\beta|+\mu \leq|\alpha|+\nu+1 \\ \mu \leq \nu}}\left\|L^{\mu} \partial^{\beta} w^{\prime}(s, \cdot)\right\|_{L^{\infty}(|x|<2)}
$$

is controlled by the right side of (3.3).

To prove this, we shall need the following

Lemma 3.3. Suppose that $w$ is as above. Suppose further that $\left(\partial_{t}^{2}-\Delta\right) w(s, y)=$ $F(s, y)=0$ if $|y|>10$. Then,

$$
(1+t) \sup _{|x|<2}\left|L^{\nu} \partial^{\alpha} w^{\prime}(t, x)\right| \leq C \sup _{0 \leq s \leq t} \sum_{\substack{|\beta|+\mu \leq \nu+|\alpha|+3 \\ \mu \leq \nu}}(1+s)\left\|L^{\mu} \partial^{\beta} F(s, \cdot)\right\|_{2} \text {. }
$$

Proof of Lemma 3.3. By Sobolev estimates, the left side of (3.8) is dominated by

$$
(1+t) \sum_{\substack{|\beta|+\mu \leq \nu+|\alpha|+2 \\ \mu \leq \nu}}\left\|L^{\mu} \partial^{\beta} w^{\prime}(t, \cdot)\right\|_{L^{2}(|x|<3)}
$$

By exponential energy decay and elliptic regularity (see Lemma 2.8 in 22]), there must be a constant $c>0$ so that this is controlled by

$$
\begin{aligned}
(1+t) \sum_{\substack{|\beta|+\mu \leq \nu+|\alpha|+1 \\
\mu \leq \nu}}\left\|L^{\mu} \partial^{\beta} F(t, \cdot)\right\|_{2}+(1+t) \int_{0}^{t} e^{-c(t-s)} \sum_{\substack {|\beta|+\mu \leq \nu+|\alpha|+3 \\
\begin{subarray}{c}{\mu \leq \nu\\
{ | \beta | + \mu \leq \nu + | \alpha | + 3 \\
\begin{subarray} { c } { \mu \leq \nu \\
} }\end{subarray}}\left\|L^{\mu} \partial^{\beta} F(s, \cdot)\right\|_{2} d s \\
\quad \leq C \sup _{\substack{0 \leq s \leq t \\
|\beta|+\mu \leq \nu+|\alpha|+3}}(1+s) \sum_{\substack{\mu \leq \nu \\
\mu}} \sum^{\beta} F(s, \cdot) \|_{2},
\end{aligned}
$$

as desired.

We also need an estimate for solutions whose forcing terms vanish near the obstacle. Suppose that $w$ is as above, but now assume that $\left(\partial_{t}^{2}-\Delta\right) w(s, y)=F(s, y)=0$ when $|y|<5$. Then, write

$$
w=w_{0}+w_{r}
$$

where $w_{0}$ solves the boundaryless wave equation $\left(\partial_{t}^{2}-\Delta\right) w_{0}=F$ with zero initial data. Fix $\eta \in C_{0}^{\infty}\left(\mathbb{R}^{3}\right)$ satisfying $\eta(x)=1$ for $|x|<2$ and $\eta(x)=0$ for $|x| \geq 3$. If we set $\tilde{w}=\eta w_{0}+w_{r}$, then since $\eta F=0, \tilde{w}$ solves the Dirichlet-wave equation

$$
\left(\partial_{t}^{2}-\Delta\right) \tilde{w}=G=-2 \nabla_{x} \eta \cdot \nabla_{x} w_{0}-(\Delta \eta) w_{0}
$$


with zero initial data. This forcing term vanishes unless $2 \leq|x| \leq 3$. In this case, by Lemma 3.3

$$
\begin{aligned}
(1+t) \sum_{|\beta|+\mu \leq|\alpha|+\nu+1} \sup _{\substack{|x|<2 \\
\mu \leq \nu}}\left|L^{\mu} \partial^{\beta} w^{\prime}(t, x)\right|=(1+t) \sum_{\substack{|\beta|+\mu \leq|\alpha|+\nu+1 \\
\mu \leq \nu}} \sup _{|x|<2}\left|L^{\mu} \partial^{\beta} \tilde{w}^{\prime}(t, x)\right| \\
\leq C \sup _{0 \leq s \leq t} \sum_{\substack{|\beta|+\mu \leq|\alpha|+\nu+4 \\
\mu \leq \nu}}(1+s)\left\|L^{\mu} \partial^{\beta} G(s, \cdot)\right\|_{2} \\
\leq C \sup _{0 \leq s \leq t} \sum_{|\beta|+\mu \leq|\alpha|+\nu+5}(1+s)\left\|L^{\mu} \partial^{\beta} w_{0}(s, \cdot)\right\|_{L^{\infty}(2<|x|<3)} .
\end{aligned}
$$

Thus, if we could show the following lemma, we would have that the (3.7) is bounded by the right side of (3.3), which would complete the proof of Theorem 3.2

Lemma 3.4. Suppose that $v$ is a solution to the free wave equation $\left(\partial_{t}^{2}-\Delta\right) v=G$ and that $v$ has vanishing Cauchy data. Suppose further that $G(t, x)=0$ when $|x|>10 t$. Then,

$$
\begin{aligned}
\sup _{2<|x|<3}|v(t, x)| \leq C \sum_{|\alpha| \leq 2} & \int_{0}^{t} \int_{\substack{|| y|-(t-s)|<10 \\
|y| \leq(600+s) / 2}}\left|\Omega^{\alpha} G(s, y)\right| \frac{d y d s}{|y|} \\
& +\frac{C}{1+t} \sum_{\substack{|\alpha|+\mu \leq 3 \\
\mu \leq 1}} \int_{t / 100}^{t} \int_{|y| \geq(1+s) / 10}\left|L^{\mu} Z^{\alpha} G(s, y)\right| \frac{d y d s}{|y|}
\end{aligned}
$$

Proof of Lemma 3.4 This lemma is a consequence of the estimate

$$
|x||v(t, x)| \leq \frac{1}{2} \int_{0}^{t} \int_{|r-(t-s)|}^{r+t-s} \sup _{|\theta|=1}|G(s, \rho \theta)| \rho d \rho d s
$$

where $r=|x|$. See, e.g., (2.4) in [14].

We begin by choosing a cutoff function $\rho(x)$ satisfying $\rho(x)=1$ for $|x|<1 / 10$ and $\rho(x)=0$ for $|x|>1 / 2$. Set $G_{1}(t, x)=\rho(x /(1+t)) G(t, x)$ and $G_{2}(t, x)=(1-\rho(x /(1+$ t)) $G(t, x)$, and for $j=1,2$, let $v_{j}$ solve the inhomogeneous wave equation $\left(\partial_{t}^{2}-\Delta\right) v_{j}=G_{j}$ with zero initial data. Then, $v=v_{1}+v_{2}$. Using (3.10) and the Sobolev estimate on the sphere, we have for $2<|x|<3$

$$
\begin{aligned}
\left|v_{1}(t, x)\right| & \leq C \sum_{|\alpha| \leq 2} \int_{0}^{t} \int_{|| y|-(t-s)|<10}\left|\Omega^{\alpha} G_{1}(s, y)\right| \frac{d y d s}{|y|} \\
& \leq C \sum_{|\alpha| \leq 2} \int_{0}^{t} \int_{\substack{|| y|-(t-s)|<10 \\
|y| \leq(1+s) / 2}}\left|\Omega^{\alpha} G(s, y)\right| \frac{d y d s}{|y|}
\end{aligned}
$$


By (3.5), if $2<|x|<3$ and $t>10|x|$,

$$
\begin{aligned}
\left|v_{2}(t, x)\right| & \leq \frac{C}{1+t} \sum_{\substack{|\alpha|+\mu \leq 3 \\
\mu \leq 1}} \int_{t / 100}^{t} \int\left|L^{\mu} Z^{\alpha} G_{2}(s, y)\right| \frac{d y d s}{|y|} \\
& \leq \frac{C}{1+t} \sum_{\substack{|\alpha|+\mu \leq 3 \\
\mu \leq 1}}^{t} \int_{t / 100}^{t} \int_{|y| \geq(1+s) / 10}\left|L^{\mu} Z^{\alpha} G(s, y)\right| \frac{d y d s}{|y|}
\end{aligned}
$$

Since $v=v_{1}+v_{2}$, these two estimates yield (3.9) when $t>10|x|$. The proof of the estimate for $v_{1}$ shows that for $0<t<10|x|$ the left side of (3.9) is dominated by the first term in the right.

4. Estimates Related to the Null Condition and Sobolev-type Estimates. The first result of this section concerns bounds for the null forms. They must involve the weight $\left\langle c_{J} t-r\right\rangle$ since we are not using the generators of Lorentz rotations. The estimates will involve the admissible homogeneous vector fields that we are using $\{\Gamma\}=\{Z, L\}$. The proof of these estimates can be found in Sideris-Tu [29] and Sogge [32.

Lemma 4.1. Suppose that the quasilinear null condition (1.9) holds. Then,

$$
\begin{aligned}
& \left|\sum_{0 \leq j, k, l \leq 3} B_{J, l}^{J J, j k} \partial_{l} u \partial_{j} \partial_{k} v\right| \\
& \leq C\langle r\rangle^{-1}\left(|\Gamma u|\left|\partial^{2} v\right|+|\partial u||\partial \Gamma v|\right)+C \frac{\left\langle c_{J} t-r\right\rangle}{\langle t+r\rangle}|\partial u|\left|\partial^{2} v\right| .
\end{aligned}
$$

Also, if the semilinear null condition (1.10) holds

$$
\left|\sum_{0 \leq j, k \leq 3} A_{J J}^{J, j k} \partial_{j} u \partial_{k} v\right| \leq C\langle r\rangle^{-1}(|\Gamma u||\partial v|+|\partial u||\Gamma v|)+C \frac{\left\langle c_{J} t-r\right\rangle}{\langle t+r\rangle}|\partial u||\partial v|
$$

We shall also need the following Sobolev-type estimate. The first is an exterior domain analog of results of Klainerman-Sideris [17.

Lemma 4.2. Suppose that $u(t, x) \in C_{0}^{\infty}\left(\mathbb{R} \times \mathbb{R}^{3} \backslash \mathcal{K}\right)$ vanishes for $x \in \partial \mathcal{K}$. Then if $|\alpha|=M$ and $\nu$ are fixed

$$
\begin{aligned}
& \left\|\langle t-r\rangle L^{\nu} Z^{\alpha} \partial^{2} u(t, \cdot)\right\|_{2} \leq C \sum_{\substack{|\beta|+\mu \leq M+\nu+1 \\
\mu \leq \nu+1}}\left\|L^{\mu} Z^{\beta} u^{\prime}(t, \cdot)\right\|_{2} \\
& +C \sum_{\substack{|\beta|+\mu \leq M+\nu \\
\mu \leq \nu}}\left\|\langle t+r\rangle L^{\mu} Z^{\beta}\left(\partial_{t}^{2}-\Delta\right) u(t, \cdot)\right\|_{2}+C(1+t) \sum_{\mu \leq \nu}\left\|L^{\mu} u^{\prime}(t, \cdot)\right\|_{L^{2}(|x|<2)} .
\end{aligned}
$$


Proof of Lemma 4.2 The first step is to show that

$$
\begin{aligned}
\left\|\langle t-r\rangle L^{\nu} Z^{\alpha} \partial^{2} u(t, \cdot)\right\|_{2} & \leq C \sum_{\substack{|\beta|+\mu \leq M+\nu+1 \\
\mu \leq \nu+1}}\left\|L^{\mu} Z^{\beta} u^{\prime}(t, \cdot)\right\|_{2} \\
& +C \sum_{\substack{|\beta|+\mu \leq M+\nu \\
\mu \leq \nu}}\left\|\langle t+r\rangle L^{\mu} Z^{\beta}\left(\partial_{t}^{2}-\Delta\right) u(t, \cdot)\right\|_{2} \\
& +C(1+t) \sum_{\substack{|\beta|+\mu \leq M+\nu+2 \\
\mu \leq \nu}}\left\|L^{\mu} \partial^{\beta} u(t, \cdot)\right\|_{L^{2}(|x|<3 / 2)}
\end{aligned}
$$

If one replaces the left side by the analogous expression with the norm taken over $|x|<3 / 2$ then this term is dominated by the last term in (4.4) due to the fact that the coefficients of $Z$ are bounded when $|x|<3 / 2$.

To handle the part where $|x|>3 / 2$ we shall use the following Minkowski space estimate

$$
\begin{aligned}
\left\|\langle t-r\rangle L^{\nu} Z^{\alpha} \partial^{2} h(t, \cdot)\right\|_{L^{2}\left(\mathbb{R}^{3}\right)} & \leq C \sum_{\substack{|\beta|+\mu \leq|\alpha|+\nu+1 \\
\mu \leq \nu+1}}\left\|L^{\mu} Z^{\beta} h^{\prime}(t, \cdot)\right\|_{L^{2}\left(\mathbb{R}^{3}\right)} \\
& +C \sum_{\substack{|\beta|+\mu \leq|\alpha|+\nu \\
\mu \leq \nu}}\left\|\langle t+r\rangle L^{\mu} Z^{\beta}\left(\partial_{t}^{2}-\Delta\right) h(t, \cdot)\right\|_{L^{2}\left(\mathbb{R}^{3}\right)},
\end{aligned}
$$

which is valid for $h \in C_{0}^{\infty}\left(\mathbb{R} \times \mathbb{R}^{3}\right)$. This estimate follows from (2.10) and Lemma 3.1 of Klainerman and Sideris [17] if one uses the fact that $\left[\left(\partial_{t}^{2}-\Delta\right), Z\right]=0$ and $\left[\left(\partial_{t}^{2}-\Delta\right), L\right]=$ $2\left(\partial_{t}-\Delta\right)$.

To use this, choose $\eta \in C_{0}^{\infty}\left(\mathbb{R}^{3}\right)$ so that $\eta(x)=0$ for $|x|<1$ and $\eta(x)=1$ for $|x|>3 / 2$. Then if we let $h(t, x)=\eta(x) u(t, x)$, we have

$$
\left(\partial_{t}^{2}-\Delta\right) h=\eta(x)\left(\partial_{t}^{2}-\Delta\right) u-2 \nabla_{x} \eta \cdot \nabla_{x} u-(\Delta \eta) u .
$$

Therefore since the last two terms are supported in $|x|<3 / 2$, (4.5) yields

$$
\begin{aligned}
\left\|\langle t-r\rangle L^{\nu} Z^{\alpha} \partial^{2} u(t, \cdot)\right\|_{L^{2}(|x|>3 / 2)} & \leq\left\|\langle t-r\rangle L^{\nu} Z^{\alpha} \partial^{2} h(t, \cdot)\right\|_{L^{2}\left(\mathbb{R}^{3}\right)} \\
& \leq C \sum_{|\beta|+\mu \leq M+\nu+1}^{\substack{\mu \leq \nu+1 \\
\mid \leq L^{\prime}}}\left\|L^{\mu} Z^{\beta} u^{\prime}(t, \cdot)\right\|_{2} \\
& +C \sum_{\substack{|\beta|+\mu \leq M+\nu \\
\mu \leq \nu}}\left\|\langle t+r\rangle L^{\mu} Z^{\beta}\left(\partial_{t}^{2}-\Delta\right) u(t, \cdot)\right\|_{2} \\
& +C(1+t) \sum_{|\beta|+\mu \leq M+\nu+1}^{\substack{\mu \leq \nu \\
\mu \leq \nu}}\left\|L^{\mu} \partial^{\beta} u(t, \cdot)\right\|_{L^{2}(|x|<3 / 2)},
\end{aligned}
$$

which completes the proof of (4.4). 
In view of this inequality, to finish the proof of the lemma we need to show that if $1<R<2$ then

$$
(1+t) \sum_{\substack{|\beta|+\mu \leq M+\nu+2 \\ \mu \leq \nu}}\left\|L^{\mu} \partial^{\beta} u(t, \cdot)\right\|_{L^{2}(|x|<R)}
$$

is controlled by the right hand side of (4.3). However, if $1<R<R_{0}<2$, by elliptic regularity this term is dominated by

$$
\begin{gathered}
(1+t)\left(\sum_{\substack{|\beta|+\mu \leq M+\nu \\
\mu \leq \nu}}\left\|L^{\mu} \partial^{\beta} \Delta u(t, \cdot)\right\|_{L^{2}\left(|x|<R_{0}\right)}+\sum_{\substack{|\beta|+\mu \leq M+\nu \\
\mu \leq \nu}}\left\|L^{\mu} \partial^{\beta} \partial_{t} u^{\prime}(t, \cdot)\right\|_{L^{2}(|x|<R)}\right. \\
\left.+\sum_{\substack{|\beta|+\mu \leq(M-1)+\nu+2 \\
\mu \leq \nu}}\left\|L^{\mu} \partial^{\beta} u(t, \cdot)\right\|_{L^{2}\left(|x|<R_{0}\right)}\right) .
\end{gathered}
$$

Since Lemma 2.3 from [17] yields

$$
\begin{gathered}
\langle t-r\rangle\left(\sum_{\substack{|\beta|+\mu \leq M+\nu \\
\mu \leq \nu}}\left|L^{\mu} \partial^{\beta} \Delta u\right|+\sum_{\substack{|\beta|+\mu \leq M+\nu \\
\mu \leq \nu}}\left|L^{\mu} \partial^{\beta} \partial_{t} u^{\prime}\right|\right) \\
\leq C \sum_{\substack{|\beta|+\mu \leq M+\nu+1 \\
\mu \leq \nu+1}}\left|L^{\mu} Z^{\beta} u^{\prime}\right|+C\langle t+r\rangle \sum_{\substack{|\beta|+\mu \leq M+\nu \\
\mu \leq \nu}}\left|L^{\mu} Z^{\beta}\left(\partial_{t}^{2}-\Delta\right) u\right|,
\end{gathered}
$$

the preceding inequality and an induction argument imply that (4.6) is dominated by the right hand side of (4.3) plus

$$
(1+t) \sum_{|\beta| \leq 1, \mu \leq \nu}\left\|L^{\mu} \partial^{\beta} u(t, \cdot)\right\|_{L^{2}(|x|<\tilde{R})},
$$

where $\tilde{R}$ can be taken to satisfy $3 / 2<\tilde{R}<2$. Since $\partial_{t}^{j} u$ vanishes on $\partial \mathcal{K}$ one can use a similar induction argument to see that this is also dominated by the right hand side of (4.3) plus

$$
(1+t) \sum_{\mu \leq \nu}\left\|L^{\mu} \nabla_{x} u(t, \cdot)\right\|_{L^{2}\left(|x|<R_{1}\right)},
$$

where $\tilde{R}<R_{1}<2$, which finishes the proof.

The next lemma is an exterior domain analog of an estimate of Hidano-Yokoyama [6].

Lemma 4.3. Suppose that $u(t, x) \in C_{0}^{\infty}\left(\mathbb{R} \times \mathbb{R}^{3} \backslash \mathcal{K}\right)$ vanishes for $x \in \partial \mathcal{K}$. Then

$$
\begin{aligned}
& \text { (4.7) } r^{1 / 2}\langle t-r\rangle\left|\partial L^{\nu} Z^{\alpha} u(t, x)\right| \leq C \sum_{\substack{|\beta|+\mu \leq|\alpha|+\nu+2 \\
\mu \leq \nu+1}}\left\|L^{\mu} Z^{\beta} u^{\prime}(t, \cdot)\right\|_{2} \\
& +C \sum_{\substack{|\beta|+\mu \leq|\alpha|+\nu+1 \\
\mu \leq \nu}}\left\|\langle t+r\rangle L^{\mu} Z^{\beta}\left(\partial_{t}^{2}-\Delta\right) u(t, \cdot)\right\|_{2}+C(1+t) \sum_{\mu \leq \nu}\left\|L^{\mu} u^{\prime}(t, \cdot)\right\|_{L^{\infty}(|x|<2)} .
\end{aligned}
$$


Proof of Lemma 4.3, Inequality (4.2) of Hidano-Yokoyama [6] implies that in Minkowski space

$$
\begin{aligned}
& r^{1 / 2}\langle t-r\rangle\left|\partial L^{\nu} Z^{\alpha} h(t, x)\right| \\
& \leq C \sum_{\substack{|\beta|+\mu \leq|\alpha|+\nu+1 \\
\mu \leq \nu}}\left(\left\|L^{\mu} Z^{\beta} h^{\prime}(t, \cdot)\right\|_{2}+\left\|\langle t-r\rangle L^{\mu} Z^{\beta} \partial^{2} h(t, \cdot)\right\|_{2}\right) .
\end{aligned}
$$

If we choose $\eta \in C_{0}^{\infty}\left(\mathbb{R}^{3}\right)$ so that $\eta(x)=0$ for $|x|<1$ and $\eta(x)=1$ for $|x|>5 / 4$, and let $h(t, x)=\eta(x) u(t, x)$, then we conclude that when $|x|>5 / 4$,

$$
\begin{aligned}
& r^{1 / 2}\langle t-r\rangle\left|\partial L^{\nu} Z^{\alpha} u(t, x)\right| \\
& \leq C \sum_{\substack{|\beta|+\mu \leq|\alpha|+\nu+1 \\
\mu \leq \nu}}\left(\left\|L^{\mu} Z^{\beta} u^{\prime}(t, \cdot)\right\|_{2}+\left\|\langle t-r\rangle L^{\mu} Z^{\beta} \partial^{2} u(t, \cdot)\right\|_{2}\right) \\
& \quad+C(1+t) \sum_{\substack{|\beta|+\mu \leq|\alpha|+\nu+3 \\
\mu \leq \nu}}\left\|L^{\mu} \partial^{\beta} u(t, \cdot)\right\|_{L^{2}(|x|<3 / 2) .} .
\end{aligned}
$$

By the Sobolev inequality, over $|x|<5 / 4$ the left side of (4.7) is bounded by a similar inequality involving only the last term on the right.

If we use (4.3), we see that the second term in the right is dominated by the right side of (4.7). If we repeat the last part of the proof of Lemma4.2 we conclude that the same is true for the last term in the preceding inequality.

Finally, we will need the following now standard consequence of the Sobolev lemma (see [16]).

Lemma 4.4. Suppose that $h \in C^{\infty}\left(\mathbb{R}^{3}\right)$. Then, for $R>2$,

$$
\|h\|_{L^{\infty}(R<|x|<R+1)} \leq C R^{-1} \sum_{|\alpha|+|\beta| \leq 2}\left\|\Omega^{\alpha} \partial_{x}^{\beta} h\right\|_{L^{2}(R-1<|x|<R+2)} .
$$

5. Global Existence and the Continuity Argument. In this section, we will prove the main result, Theorem 1.1. We shall take $N=101$ in its smallness hypothesis (1.11), but this is certainly not optimal.

To prove our global existence theorem, we shall need a standard local existence theorem.

Theorem 5.1. Suppose that $f$ and $g$ are as in Theorem 1.1 with $N \geq 6$ in (1.11). Then there is a $T>0$ so that the initial value problem (1.5) with this initial data has a $C^{2}$ solution satisfying

$$
u \in L^{\infty}\left([0, T] ; H^{N}\left(\mathbb{R}^{3} \backslash \mathcal{K}\right)\right) \cap C^{0,1}\left([0, T] ; H^{N-1}\left(\mathbb{R}^{3} \backslash \mathcal{K}\right)\right) .
$$

The supremum of such $T$ is equal to the supremum of all $T$ such that the initial value problem has a $C^{2}$ solution with $\partial^{\alpha} u$ bounded for $|\alpha| \leq 2$. Also, one can take $T \geq 2$ if $\|f\|_{H^{N}}+\|g\|_{H^{N-1}}$ is sufficiently small.

This essentially follows from the local existence results Theorem 9.4 and Lemma 9.6 in [12. The latter were only stated for diagonal single-speed systems; however, since the 
proof relied only on energy estimates, it extends to the multi-speed non-diagonal case if the symmetry assumptions (1.8) are satisfied.

Next, as in [14], in order to avoid dealing with compatibility conditions for the Cauchy data, it is convenient to reduce the Cauchy problem (1.5) to an equivalent equation with a nonlinear driving force but vanishing Cauchy data. We will then set up a continuity argument that utilizes the results of the previous three sections to show global existence and prove Theorem 1.1

Recall that our smallness condition on the data is

$$
\sum_{|\alpha| \leq 101}\left\|<x>^{|\alpha|} \partial_{x}^{\alpha} f\right\|_{L^{2}\left(\mathbb{R}^{3} \backslash \mathcal{K}\right)}+\sum_{|\alpha| \leq 100}\left\|<x>^{1+|\alpha|} \partial_{x}^{\alpha} g\right\|_{L^{2}\left(\mathbb{R}^{3} \backslash \mathcal{K}\right)} \leq \varepsilon .
$$

To make the reduction to an equation with vanishing initial data, we will begin by noting that if the data satisfies (5.1) for $\varepsilon$ sufficiently small, then we can find a solution $u$ to (1.5) on a set of the form $0<c t<|x|$ where $c=5 \max _{I} c_{I}$, and this solution satisfies

$$
\sup _{0<t<\infty} \sum_{|\alpha| \leq 101}\left\|<x>^{|\alpha|} \partial^{\alpha} u(t, \cdot)\right\|_{L^{2}\left(\mathbb{R}^{3} \backslash \mathcal{K}:|x|>c t\right)} \leq C_{0} \varepsilon,
$$

for an absolute constant $C_{0}$.

To prove this, we shall repeat the argument of Keel-Smith-Sogge [14. By scaling in $t$, we may assume without loss that $\max _{I} c_{I}=1 / 2$. Theorem [5.1]yields a solution $u$ to (1.5) on the set $0<t<2$ which satisfies (5.2). We wish to show that this solution extends to the set $0<c t<|x|$. To do so, let $R \geq 4$ and consider data $\left(f_{R}, g_{R}\right)$ supported in the set $R / 4<|x|<4 R$ which agrees with the data $(f, g)$ on the set $R / 2<|x|<2 R$. Let $u_{R}(t, x)$ satisfy the free wave equation

$$
\square u_{R}=Q\left(d u_{R}, R^{-1} d^{2} u_{R}\right)
$$

with Cauchy data $\left(f_{R}(R \cdot), R g_{R}(R \cdot)\right)$. The solution $u_{R}$ then exists for $0<t<1$ by standard local existence theory (see, e.g., 7] and [31]) and satisfies

$$
\begin{aligned}
\sup _{0<t<1}\left\|u_{R}(t, \cdot)\right\|_{H^{101}\left(\mathbb{R}^{3}\right)} \leq C\left(\left\|f_{R}(R \cdot)\right\|_{H^{101}\left(\mathbb{R}^{3}\right)}+R\left\|g_{R}(R \cdot)\right\|_{H^{100}\left(\mathbb{R}^{3}\right)}\right) \\
\leq C R^{-3 / 2}\left(\sum_{|\alpha| \leq 101}\left\|\left(R \partial_{x}\right)^{\alpha} f_{R}\right\|_{L^{2}\left(\mathbb{R}^{3}\right)}+R \sum_{|\alpha| \leq 100}\left\|\left(R \partial_{x}\right)^{\alpha} g_{R}\right\|_{L^{2}\left(\mathbb{R}^{3}\right)}\right) .
\end{aligned}
$$

The smallness condition on $\left|u_{R}^{\prime}\right|$ implies that the wave speeds for the quasilinear equation are bounded above by 1 . A domain of dependence argument shows that the solutions $u_{R}\left(R^{-1} t, R^{-1} x\right)$ restricted to ||$x|-R|<\frac{R}{2}-t$ agree on their overlaps, and also with the local solution, yielding a solution to (1.5) on the set $\left\{\mathbb{R}^{3} \backslash \mathcal{K}: 2 t<|x|\right\}$. An argument using a partition of unity now yields (5.2).

We are now ready to set up the continuity argument. We will use the local solution $u$ to allow us to restrict to the case where the Cauchy data vanish. Fix a cutoff function $\chi \in C^{\infty}(\mathbb{R})$ satisfying $\chi(s)=1$ if $s \leq \frac{1}{2 c}$ and $\chi(s)=0$ if $s>\frac{1}{c}$. Set

$$
u_{0}(t, x)=\eta(t, x) u(t, x), \quad \eta(t, x)=\chi\left(|x|^{-1} t\right) .
$$

Assuming as we may that $0 \in \mathcal{K}$, we have that $|x|$ is bounded below on the complement of $\mathcal{K}$ and the function $\eta(t, x)$ is smooth and homogeneous of degree 0 in $(t, x)$. Additionally,

$$
\square u_{0}=\eta Q\left(d u, d^{2} u\right)+[\square, \eta] u \text {. }
$$


Thus, $u$ solves $\square u=Q\left(d u, d^{2} u\right)$ for $0<t<T$ if and only if $w=u-u_{0}$ solves

$$
\left\{\begin{array}{l}
\square w=(1-\eta) Q\left(d u, d^{2} u\right)-[\square, \eta] u \\
\left.w\right|_{\partial \mathcal{K}}=0 \\
w(t, x)=0, \quad t \leq 0
\end{array}\right.
$$

for $0<t<T$.

A key step in proving that (5.3) admits a global solution is to prove uniform energy and dispersive estimates for $w$ on the interval of existence. First note that since $u_{0}=\eta u$, by (5.2) and Lemma 4.4 there is an absolute constant $C_{1}$ so that

$$
\begin{aligned}
& (1+t+|x|) \sum_{\mu+|\alpha| \leq 99}\left|L^{\mu} Z^{\alpha} u_{0}(t, x)\right| \\
& \quad+\sum_{\mu+|\alpha|+|\beta| \leq 101}\left\|<t+r>^{|\beta|} L^{\mu} Z^{\alpha} \partial^{\beta} u_{0}(t, \cdot)\right\|_{2} \leq C_{1} \varepsilon
\end{aligned}
$$

Furthermore, if we let $v$ be the solution of the linear equation

$$
\left\{\begin{array}{l}
\square v=-[\square, \eta] u \\
\left.v\right|_{\partial \mathcal{K}}=0 \\
v(t, x)=0, \quad t \leq 0
\end{array}\right.
$$

then we will show that (5.2) and Theorem 3.1 imply that there is an absolute constant $C_{2}$ so that

$$
(1+t+|x|) \sum_{\mu+|\alpha| \leq 92}\left|L^{\mu} Z^{\alpha} v(t, x)\right|+\sum_{\mu+|\alpha| \leq 90}\left\|L^{\mu} Z^{\alpha} v^{\prime}(t, \cdot)\right\|_{2} \leq C_{2} \varepsilon .
$$

Indeed, by (3.2), the first term on the left side of (5.6) is bounded by

$$
\begin{aligned}
\int_{0}^{t} \int_{|x|>c s} \sum_{\mu+|\alpha| \leq 99}\left|L^{\mu} Z^{\alpha}([\square, \eta] u)(s, x)\right| & \frac{d x d s}{|x|} \\
& +\int_{0}^{t} \sum_{\mu+|\alpha| \leq 96}\left\|L^{\mu} \partial^{\beta}([\square, \eta] u)(s, \cdot)\right\|_{L^{2}\left(\mathbb{R}^{3} \backslash \mathcal{K}:|x|<2\right)} d s
\end{aligned}
$$

which by the Schwarz inequality is bounded by

$$
\sum_{\mu+|\alpha| \leq 99} \sum_{j=0}^{\infty} \sup _{0<c s<2^{j+1}}\left\|<x>^{3 / 2} L^{\mu} Z^{\alpha}[\square, \eta] u(s, \cdot)\right\|_{L^{2}\left(\mathbb{R}^{3} \backslash \mathcal{K}: 2^{j}<|x|<2^{j+1}\right)} .
$$

Since this is dominated by

$$
\sup _{0<t<\infty} \sum_{\mu+|\alpha| \leq 99}\left\|<x>^{2} L^{\mu} Z^{\alpha}[\square, \eta] u(t, \cdot)\right\|_{2},
$$

one gets that the first term on the left side of (5.6) is $O(\varepsilon)$ from (5.2) and the homogeneity of $\eta$. 
For the second term on the left side of (5.6), if we argue as in the proof of (2.5) (except now for the linear wave equation), we see that

$$
\begin{aligned}
\partial_{t} \sum_{\mu+|\alpha| \leq 90}\left\|L^{\mu} Z^{\alpha} v^{\prime}(t, \cdot)\right\|_{2}^{2} & \\
& \leq C\left(\sum_{\mu+|\alpha| \leq 90}\left\|L^{\mu} Z^{\alpha} v^{\prime}(t, \cdot)\right\|_{2}\right)\left(\sum_{\mu+|\alpha| \leq 90}\left\|L^{\mu} Z^{\alpha} \square v(t, \cdot)\right\|_{2}\right) \\
& +C \sum_{\mu+|\alpha| \leq 90}\left|\int_{\partial \mathcal{K}} \partial_{0} L^{\mu} Z^{\alpha} v(t, \cdot) \nabla L^{\mu} Z^{\alpha} v(t, \cdot) \cdot n d \sigma\right|,
\end{aligned}
$$

where $n$ is the outward normal at a given point on $\partial \mathcal{K}$. Since $\mathcal{K} \subset\{|x|<1\}$, it follows that

$$
\begin{aligned}
\partial_{t} \sum_{\mu+|\alpha| \leq 90}\left\|L^{\mu} Z^{\alpha} v^{\prime}(t, \cdot)\right\|_{2}^{2} & \\
& \leq C\left(\sum_{\mu+|\alpha| \leq 90}\left\|L^{\mu} Z^{\alpha} v^{\prime}(t, \cdot)\right\|_{2}\right)\left(\sum_{\mu+|\alpha| \leq 90}\left\|L^{\mu} Z^{\alpha} \square v(t, \cdot)\right\|_{2}\right) \\
& +C \int_{\left\{x \in \mathbb{R}^{3} \backslash \mathcal{K}:|x|<1\right\}} \sum_{\mu+|\alpha| \leq 91}\left|L^{\mu} Z^{\alpha} v^{\prime}(t, \cdot)\right|^{2} d x .
\end{aligned}
$$

Thus, since $\square v(s, y)=-[\square, \eta] u(s, y)$, it follows that

$$
\begin{aligned}
\sum_{\mu+|\alpha| \leq 90}\left\|L^{\mu} Z^{\alpha} v^{\prime}(t, \cdot)\right\|_{2}^{2} \leq C\left(\int_{0}^{t}\right. & \left.\sum_{\mu+|\alpha| \leq 90}\left\|L^{\mu} Z^{\alpha}(-[\square, \eta] u)(s, y)\right\|_{2} d s\right)^{2} \\
& +C \int_{0}^{t} \sum_{\mu+|\alpha| \leq 91}\left\|L^{\mu} Z^{\alpha} v^{\prime}(s, \cdot)\right\|_{L^{2}(|x|<1)}^{2} d s .
\end{aligned}
$$

The first term on the right is $O(\varepsilon)$ by (5.2). Using the bound for the first term in the left of (5.6), it follows that the second term on the right of (5.7) is also $O(\varepsilon)$ as desired.

Using this, we are now ready to set up the continuity argument. If $\varepsilon>0$ is as above, we shall assume that we have a solution of our equation (1.5) for $0 \leq t \leq T$ such that we 
have the following estimates

$$
\begin{aligned}
& \sum_{\substack{|\alpha|+\nu \leq 52 \\
\nu \leq 2}}\left\|L^{\nu} Z^{\alpha} w^{\prime}(t, \cdot)\right\|_{2} \leq A_{0} \varepsilon \\
& (1+t+r) \sum_{|\alpha| \leq 40}\left|Z^{\alpha} w^{\prime}(t, x)\right| \leq A_{1} \varepsilon \\
& (1+t+r) \sum_{\substack{|\alpha|+\nu \leq 55 \\
\nu \leq 3}}\left|L^{\nu} Z^{\alpha}(w-v)(t, x)\right| \leq B_{1} \varepsilon^{2}(1+t)^{1 / 10} \log (2+t) \\
& \sum_{|\alpha| \leq 100}\left\|\partial^{\alpha} u^{\prime}(t, \cdot)\right\|_{2} \leq B_{2} \varepsilon(1+t)^{1 / 40} \\
& \sum_{\substack{|\alpha|+\nu \leq 65 \\
\nu \leq 4}}\left\|L^{\nu} Z^{\alpha} u^{\prime}(t, \cdot)\right\|_{2} \leq B_{3} \varepsilon(1+t)^{1 / 20} \\
& \sum_{\substack{|\alpha|+\nu \leq 63 \\
\nu \leq 4}}\left\|\langle x\rangle^{-1 / 2} L^{\nu} Z^{\alpha} u^{\prime}\right\|_{L^{2}\left(S_{t}\right)} \leq B_{4} \varepsilon(1+t)^{1 / 20}(\log (2+t))^{1 / 2} .
\end{aligned}
$$

Here, as before, the $L^{2}$ norms are taken over $\mathbb{R}^{3} \backslash \mathcal{K}$ and the weighted $L_{t}^{2} L_{x}^{2}$ norms are taken over $S_{t}=[0, t] \times \mathbb{R}^{3} \backslash \mathcal{K}$. In the main estimates (5.8) and (5.9), we can take $A_{0}=A_{1}=4 C_{2}$, where $C_{2}$ is the constant occurring in the bounds (5.6) for $v$.

Clearly if $\varepsilon$ is small then all of these estimates are valid, if $T=2$, by Theorem [5.1] Keeping this in mind, we shall then prove that, for $\varepsilon>0$ smaller than some number depending on $B_{1}, \ldots, B_{4}$,

i.) (5.8) is valid with $A_{0}$ replaced by $A_{0} / 2$.

ii.) Under the assumption of (i.), that (5.9) is valid with $A_{1}$ replaced by $A_{1} / 2$.

iii.) (5.10)-(5.13) are consequences of (5.8) and (5.9) for suitable constants $B_{i}$.

By the local existence theorem, it will follow that a solutions exists for all $t>0$ if $\varepsilon$ is small enough.

Before we begin the proof of (i.), we will set up some preliminary results under the assumption of (5.8)-(5.13). That is, we wish to show that

$$
r^{1 / 2}\langle t-r\rangle\left|L^{\nu} Z^{\alpha} u^{\prime}(t, x)\right| \leq C \varepsilon(1+t)^{3 / 20} \log (2+t)
$$

and

$$
\left\|\langle t+r\rangle L^{\nu} Z^{\alpha} \square u(t, \cdot)\right\|_{2} \leq C \varepsilon(1+t)^{3 / 20} \log (2+t)
$$

for $\nu \leq 2$ and $|\alpha|+\nu \leq 63$. Notice that the first follows from the second by (4.7), (5.4), (5.6), (5.10), and (5.12). For (5.15), we expand $\square u$ according to (1.7) to see that the left side is dominated by

$$
\left\|\left(\langle t+r\rangle \sum_{\substack{|\alpha|+\mu \leq 32 \\ \mu \leq 2}}\left|L^{\mu} Z^{\alpha} u^{\prime}(t, \cdot)\right|\right) \sum_{\substack{|\alpha|+\mu \leq 64 \\ \mu \leq 2}}\left|L^{\mu} Z^{\alpha} u^{\prime}(t, \cdot)\right|\right\|_{2} .
$$

By (5.10) and (5.12), this is easily seen to be bounded by the right side of (5.15) as desired. 
Since $|\square(w-v)| \leq C|\square u|$, it is clear that the same proof also yields

$$
r^{1 / 2}\langle t-r\rangle\left|L^{\nu} Z^{\alpha}(w-v)^{\prime}(t, x)\right| \leq C \varepsilon(1+t)^{3 / 20} \log (2+t) .
$$

Let's begin with (i.). Since $v$ satisfies the better bound (5.6), it suffices to show

$$
\sum_{\substack{|\alpha|+\nu \leq 52 \\ \nu \leq 2}}\left\|L^{\nu} Z^{\alpha}(w-v)^{\prime}(t, \cdot)\right\|_{2}^{2} \leq C \varepsilon^{3} .
$$

By the standard energy integral method (see, e.g., Sogge [31, p.12), we have that the left side of (5.17) is bounded by

$$
\begin{aligned}
C \sum_{\substack{|\alpha|+\nu \leq 52 \\
\nu \leq 2}} \int_{0}^{t} \int_{\mathbb{R}^{3} \backslash \mathcal{K}} & \left|\left\langle\partial_{0} L^{\nu} Z^{\alpha}(w-v), \square L^{\nu} Z^{\alpha}(w-v)\right\rangle\right| d y d s \\
& +C \sum_{\substack{|\alpha|+\nu \leq 52 \\
\nu \leq 2}}\left|\int_{0}^{t} \sum_{a=1}^{3} \int_{\partial \mathcal{K}} \partial_{0} L^{\nu} Z^{\alpha}(w-v) \partial_{a} L^{\nu} Z^{\alpha}(w-v) n_{a} d \sigma d s\right|
\end{aligned}
$$

where $n=\left(n_{1}, n_{2}, n_{3}\right)$ is the outward normal at a given point on $\partial \mathcal{K}$ and $\langle\cdot, \cdot \cdot\rangle$ is the standard Euclidean inner product on $\mathbb{R}^{D}$. Since $\mathcal{K} \subset\{|x|<1\}$ and since $\mid L^{\nu} Z^{\alpha}(w-$ $v)^{\prime}(t, x)\left|\leq C \sum_{|\beta| \leq|\alpha|, \mu \leq \nu}\right| L^{\mu} \partial^{\beta}(w-v)^{\prime}(t, x) \mid$ for $x \in \partial \mathcal{K}$, we have that the last term is bounded by

$$
C \int_{0}^{t} \int_{\left\{x \in \mathbb{R}^{3} \backslash \mathcal{K}:|x|<1\right\}} \sum_{\substack{|\alpha|+\nu \leq 53 \\ \nu \leq 2}}\left|L^{\nu} \partial^{\alpha}(w-v)^{\prime}(s, y)\right|^{2} d y d s
$$

Since we also have that $[\square, L]=2 \square$ and $[\square, Z]=0$ and that $\square(w-v)=(1-\eta) \square u=$ $(1-\eta) Q\left(d u, d^{2} u\right)$, we see that the left side of (5.17) is thus controlled by

$$
\begin{aligned}
C \int_{0}^{t} \int_{\mathbb{R}^{3} \backslash \mathcal{K}}\left|\left\langle\sum_{\substack{|\alpha|+\nu \leq 52 \\
\nu \leq 2}} \partial_{0} L^{\nu} Z^{\alpha}(w-v), \sum_{\substack{|\alpha|+\nu \leq 52 \\
\nu \leq 2}} L^{\nu} Z^{\alpha} Q\left(d u, d^{2} u\right)\right\rangle\right| d y d s \\
+C \int_{0}^{t} \int_{\left\{x \in \mathbb{R}^{3} \backslash \mathcal{K}:|x|<1\right\}} \sum_{\substack{|\alpha|+\nu \leq 53 \\
\nu \leq 2}}\left|L^{\nu} \partial^{\alpha}(w-v)^{\prime}(s, y)\right|^{2} d y d s .
\end{aligned}
$$


By (1.7), this is dominated by:

$$
\begin{aligned}
& C \int_{0}^{t} \int_{\mathbb{R}^{3} \backslash \mathcal{K}} \mid \sum_{K=1}^{D} \sum_{\substack{|\alpha|+\nu \leq 52 \\
\nu \leq 2}} \partial_{0} L^{\nu} Z^{\alpha}(w-v)^{K} \sum_{0 \leq j, k, l \leq 3} B_{K, l}^{K K, j k} \sum_{\substack{|\alpha|+\nu \leq 52 \\
\nu \leq 2}} \partial_{l} L^{\nu} Z^{\alpha} u^{K} \\
& \times \sum_{\substack{|\alpha|+\nu \leq 52 \\
\nu \leq 2}} \partial_{j} \partial_{k} L^{\nu} Z^{\alpha} u^{K} \mid d y d s
\end{aligned}
$$

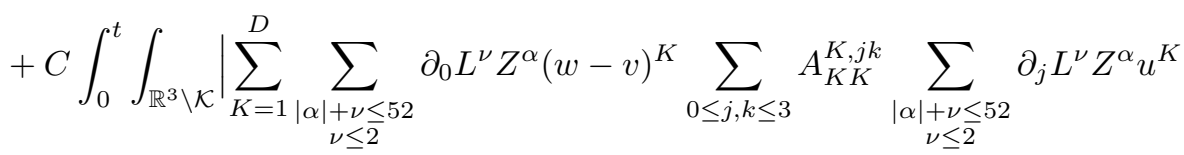

$$
\begin{aligned}
& \times \sum_{\substack{|\alpha|+\nu \leq 52 \\
\nu \leq 2}} \partial_{k} L^{\nu} Z^{\alpha} u^{K} \mid d y d s \\
& +C \int_{0}^{t} \int_{\mathbb{R}^{3} \backslash \mathcal{K}} \sum_{\substack{1 \leq I, J, K \leq D \\
(I, K) \neq(K, J)}} \sum_{\substack{|\alpha|+\nu \leq 52 \\
\nu \leq 2}}\left|L^{\nu} Z^{\alpha} \partial(w-v)^{K}\right| \sum_{\substack{|\alpha|+\nu \leq 52 \\
\nu \leq 2}}\left|L^{\nu} Z^{\alpha} \partial u^{I}\right| \\
& \times \sum_{\substack{|\alpha|+\nu \leq 53 \\
\nu \leq 2}}\left|L^{\nu} Z^{\alpha} \partial u^{J}\right| d y d s \\
& +C \int_{0}^{t} \int_{\left\{x \in \mathbb{R}^{3} \backslash \mathcal{K}:|x|<1\right\}} \sum_{\substack{|\alpha|+\nu \leq 53 \\
\nu \leq 2}}\left|L^{\mu} \partial^{\alpha}(w-v)^{\prime}(s, y)\right|^{2} d y d s .
\end{aligned}
$$

The first two terms in (5.18) satisfy the bounds of Lemma4.1 The third term involves interactions between waves of different speeds.

When dealing with the first three terms of (5.18), depending on the linear estimates we shall employ, at times we shall use certain $L^{2}$ and $L^{\infty}$ bounds for $u$ while at other times, we shall use them for $w-v$. Since $u=(w-v)+v+u_{0}$ and $u_{0}, v$ satisfy the bounds (5.4), (5.6) respectively, it will always be the case that bounds for $w-v$ will imply those for $u$ and vice versa.

Let us first handle the null terms. By (4.1) and (4.2), the first two terms in (5.18) are controlled by

$$
\begin{gathered}
C \int_{0}^{t} \int_{\mathbb{R}^{3} \backslash \mathcal{K}} \sum_{\substack{|\alpha|+\mu \leq 54 \\
\mu \leq 3}}\left|L^{\mu} Z^{\alpha} u\right| \sum_{\substack{|\alpha|+\mu \leq 54 \\
\mu \leq 3}}\left|L^{\mu} Z^{\alpha} u^{\prime}\right| \sum_{\substack{|\alpha|+\mu \leq 52 \\
\mu \leq 2}}\left|L^{\mu} Z^{\alpha}(w-v)^{\prime}\right| \frac{d y d s}{|y|} \\
+C \int_{0}^{t} \int_{\mathbb{R}^{3} \backslash \mathcal{K}} \sum_{J=1}^{D} \frac{\left\langle c_{J} s-r\right\rangle}{\langle s+r\rangle} \sum_{\substack{|\alpha|+\mu \leq 52 \\
\mu \leq 2}}\left|L^{\mu} Z^{\alpha} \partial(w-v)\right|\left(\sum_{\substack{|\alpha|+\mu \leq 53 \\
\mu \leq 2}}\left|L^{\mu} Z^{\alpha} \partial u\right|\right)^{2} d y d s
\end{gathered}
$$


To handle the contribution of the first term of (5.19), notice that by (5.4), (15.6), and (5.10) we have

$$
\sum_{\substack{|\alpha|+\mu \leq 54 \\ \mu \leq 3}}\left|L^{\mu} Z^{\alpha} u(s, y)\right| \leq C \varepsilon\langle s+|y|\rangle^{-9 / 10} \log (2+s),
$$

which means that the first term of (5.19) has a contribution to (5.18) which is dominated by

$$
\begin{gathered}
C \varepsilon \int_{0}^{t} \frac{\log (2+s)}{\langle s\rangle^{9 / 10}} \sum_{\substack{|\alpha|+\mu \leq 54 \\
\mu \leq 3}}\left\|\langle y\rangle^{-1 / 2} L^{\mu} Z^{\alpha} u^{\prime}(s, y)\right\|_{2} \\
\times \sum_{\substack{|\alpha|+\mu \leq 52 \\
\mu \leq 2}}\left\|\langle y\rangle^{-1 / 2} L^{\mu} Z^{\alpha}(w-v)^{\prime}(s, y)\right\|_{2} d s
\end{gathered}
$$

by the Schwarz inequality. Thus, if we again apply the Schwarz inequality and (5.13), we see that this contribution is $O\left(\varepsilon^{3}\right)$.

We now want to show that the second term of (5.19) satisfies a similar bound. If we apply (5.16), we see that the second term of (5.19) is controlled by

$$
\begin{gathered}
\text { (5.20) CE } \int_{0}^{t}(1+s)^{3 / 20} \log (2+s) \int_{\mathbb{R}^{3} \backslash \mathcal{K}} \frac{1}{r^{1 / 2}\langle s+r\rangle} \sum_{\substack{|\alpha|+\mu \leq 53 \\
\mu \leq 2}}\left|L^{\mu} Z^{\alpha} \partial u\right|^{2} d y d s \\
\leq C \varepsilon \int_{0}^{t} \frac{\log (2+s)}{(1+s)^{27 / 20}} \sum_{\substack{|\alpha|+\mu \leq 53 \\
\mu \leq 2}}|| L^{\mu} Z^{\alpha} u^{\prime}(s, \cdot) \|_{L^{2}(|y|>s / 2)}^{2} d s \\
+C \varepsilon \int_{0}^{t}(1+s)^{3 / 20} \log (2+s) \int_{\mathbb{R}^{3} \backslash \mathcal{K},|y| \leq s / 2} \frac{1}{r^{1 / 2}\langle s+r\rangle} \sum_{|\alpha|+\mu \leq 53}\left|L^{\mu} Z^{\alpha} u^{\prime}(s, y)\right|^{2} d y d s .
\end{gathered}
$$

The first term on the right of (5.20) is clearly $O\left(\varepsilon^{3}\right)$ by (5.12). For the second term on the right of (5.20), we apply (5.14) to control it as follows

$$
C \varepsilon^{2} \int_{0}^{t} \frac{1}{\langle s\rangle^{(6 / 5)-2 \delta}} \int_{\mathbb{R}^{3} \backslash \mathcal{K},|y| \leq s / 2} \frac{1}{r^{(3 / 2)+\delta}} \sum_{\substack{|\alpha|+\mu \leq 53 \\ \mu \leq 2}}\left|L^{\mu} Z^{\alpha} u^{\prime}(s, y)\right| d y d s .
$$

Thus, if $\delta$ is sufficiently small, the Schwarz inequality and (5.12) show that this term is also $O\left(\varepsilon^{3}\right)$. This concludes the proof that the contribution of the null forms enjoys an $O\left(\varepsilon^{3}\right)$ bound.

We now wish to show that the multi-speed terms

$$
\int_{0}^{t} \int_{\mathbb{R}^{3} \backslash \mathcal{K}} \sum_{\substack{|\alpha|+\mu \leq 52 \\ \mu \leq 2}}\left|\partial L^{\mu} Z^{\alpha}(w-v)^{K}\right| \sum_{\substack{|\alpha|+\mu \leq 52 \\ \mu \leq 2}}\left|\partial L^{\mu} Z^{\alpha} u^{I}\right| \sum_{\substack{|\alpha|+\mu \leq 53 \\ \mu \leq 2}}\left|\partial L^{\mu} Z^{\alpha} u^{J}\right| d y d s
$$

with $(I, K) \neq(K, J)$ have the same contribution to (5.18). For simplicity, let us assume that $I \neq K, I=J$. A symmetric argument will yield the same bound for the remaining cases. If we set $\delta<\left|c_{I}-c_{K}\right| / 2$, it follows that $\left\{|y| \in\left[(1-\delta) c_{I} s,(1+\delta) c_{I} s\right]\right\} \cap\{|y| \in$ 
$\left.\left[(1-\delta) c_{K} s,(1+\delta) c_{K} s\right]\right\}=\emptyset$. Thus, it will suffice to show the bound when the spatial integral is taken over the complements each of these sets separately. We will show the bound over $\left\{|y| \notin\left[(1-\delta) c_{K} s,(1+\delta) c_{K} s\right]\right\}$. The same argument will symmetrically yield the bound over the other set.

If we apply (5.16), we see that over $\left\{|y| \notin\left[(1-\delta) c_{K} s,(1+\delta) c_{K} s\right]\right\}$ (5.21) is bounded by

$$
\varepsilon \int_{0}^{t} \frac{\log (2+s)}{\langle s\rangle^{17 / 20}} \int_{\mathbb{R}^{3} \backslash \mathcal{K},|y| \notin\left[(1-\delta) c_{K} s,(1+\delta) c_{K} s\right]} \frac{1}{r^{1 / 2}} \sum_{\substack{|\alpha|+\mu \leq 53 \\ \mu \leq 2}}\left|\partial L^{\mu} Z^{\alpha} u^{I}\right|^{2} d y d s .
$$

Arguing as above, it is easy to see that these multiple speed terms are also $O\left(\varepsilon^{3}\right)$.

Finally, we need to show that the last term in (5.18) enjoys an $O\left(\varepsilon^{4}\right)$ contribution. This is clear, however, since this term is bounded by

$$
\int_{0}^{t} \sum_{\substack{|\alpha|+\mu \leq 53 \\ \mu \leq 2}}\left\|L^{\mu} \partial^{\alpha}(w-v)^{\prime}(s, \cdot)\right\|_{\infty}^{2} d s .
$$

An application of (5.10) yields the desired bounds and completes the proof of (i.).

We are now ready to prove (ii). That is, we want to show that we can prove (5.9) with $A_{1}$ replaced by $A_{1} / 2$. In view of the bounds (5.6), we see that it suffices to prove

$$
(1+t+r) \sum_{|\alpha| \leq 40}\left|Z^{\alpha}(w-v)^{\prime}(t, x)\right| \leq C \varepsilon^{3 / 2} .
$$

The estimate is straightforward when $|x|>t / 10$. For then if we use Lemma 4.4 and (5.17), we get

$$
\begin{aligned}
(1+t+|x|) \sum_{|\alpha| \leq 40}\left|Z^{\alpha}(w-v)^{\prime}(t, x)\right| & \leq C \sum_{|\alpha| \leq 42}\left\|Z^{\alpha}(w-v)^{\prime}(t, \cdot)\right\|_{2} \\
& \leq C \varepsilon^{3 / 2}, \quad|x|>t / 10 .
\end{aligned}
$$

On account of this we only need to estimate the left side of (5.23) when $|x|<t / 10$. Notice that $\square(w-v)=(1-\eta) Q\left(d u, d^{2} u\right)$ vanishes when $|x|>10 t$. Thus, we can apply (3.3) to conclude that when $|x|<t / 10$, the left side of (5.23) is dominated by

$$
\begin{aligned}
& \sum_{\substack{|\beta|+\mu \leq 43 \\
\mu \leq 1}} \int_{t / 100}^{t} \int\left|L^{\mu} Z^{\beta} \partial\left[(1-\eta) Q\left(d u, d^{2} u\right)\right]\right| \frac{d y d s}{|y|} \\
& +C \sup _{0 \leq s \leq t}(1+s) \sum_{|\beta| \leq 44}\left\|Z^{\beta}\left[(1-\eta) Q\left(d u, d^{2} u\right)\right](s, \cdot)\right\|_{\infty} \\
& +C \sup _{0 \leq s \leq t}(1+s) \sum_{|\beta| \leq 47} \int_{0}^{s} \int_{|| y|-(s-\tau)|<10}\left|Z^{\beta}\left[(1-\eta) Q\left(d u, d^{2} u\right)\right](\tau, y)\right| \frac{d y d \tau}{|y|} \\
& +C \sup _{0 \leq s \leq t} \sum_{|\beta|+\mu \leq 48} \frac{1}{1+s} \int_{0}^{s} \int_{|y| \geq(1+\tau) / 10}\left|L^{\mu} Z^{\beta}\left[(1-\eta) Q\left(d u, d^{2} u\right)\right](\tau, y)\right| d y d \tau \\
& =I+I I+I I I+I V .
\end{aligned}
$$


Terms $I I$ and $I V$ are the easiest to handle. Since $u=(w-v)+v+u_{0}$, by using (5.4), (5.6), and (5.10), one finds that $I I$ is $O\left(\varepsilon^{2}\right)$. Additionally, since (5.4), (5.6), and (5.8) yield

$$
\sum_{\substack{|\beta|+\mu \leq 49 \\ \mu \leq 1}}\left\|L^{\mu} Z^{\beta} u^{\prime}(\tau, \cdot)\right\|_{2} \leq C \varepsilon
$$

we can conclude that $I V$ is also $O\left(\varepsilon^{2}\right)$.

Similar considerations imply that

$$
I \leq \int_{t / 100}^{t} \int_{|y|<s / 2} \sum_{\substack{|\beta|+\mu \leq 43 \\ \mu \leq 1}}\left|L^{\mu} Z^{\beta} \partial Q\left(d u, d^{2} u\right)(s, y)\right| \frac{d y d s}{|y|}+C \varepsilon^{2}
$$

since $|y|^{-1}=O(1 / t)$ when $|y|>s / 2$ and $t / 100<s<t$ and since $\partial \eta=O(1 / t)$ when $t / 100<s<t$. The first term on the right side of (15.25) is dominated by

$$
\int_{t / 100}^{t} \int_{|y|<s / 2} \sum_{\substack{|\beta|+\mu \leq 44 \\ \mu \leq 1}}\left|r^{1 / 2}\langle s-r\rangle L^{\mu} Z^{\beta} u^{\prime}\right| \sum_{\substack{|\beta|+\mu \leq 44 \\ \mu \leq 1}}\left|r\langle s-r\rangle L^{\mu} Z^{\beta} u^{\prime \prime}\right| \frac{d y d s}{|y|^{3} s^{3 / 2}}
$$

If we apply Lemma 4.4 and (4.3), we see that

$$
\sum_{\substack{|\beta| \leq 44 \\ \mu \leq 1}}\left|r\langle s-r\rangle L^{\mu} Z^{\beta} u^{\prime \prime}(s, y)\right| \leq C \varepsilon(1+s)^{3 / 20} \log (2+s)
$$

by (5.4), (5.6), (5.8), and (5.15). Thus, it follows that (5.26), and hence $I$, is also $O\left(\varepsilon^{2}\right)$ using (5.14) and (5.27).

It remains to estimate $I I I$. If we use (4.7), we conclude that on the region of integration

$$
\begin{aligned}
& \sum_{|\beta| \leq 48}\left|Z^{\beta} u^{\prime}(\tau, y)\right| \\
& \leq \frac{C}{r^{1 / 2} \tau}\left[\sum_{\substack{|\beta|+\mu \leq 50 \\
\mu \leq 1}}\left\|L^{\mu} Z^{\beta} u^{\prime}(\tau, \cdot)\right\|_{2}+\sum_{|\beta| \leq 49}\left\|\langle\tau+r\rangle Z^{\beta} \square u(\tau, \cdot)\right\|_{2}+(1+\tau)\left\|u^{\prime}(\tau, \cdot)\right\|_{\infty}\right] \\
& \leq \frac{C}{r^{1 / 2} \tau}\left[\varepsilon+\sum_{|\beta| \leq 49}\left\|\langle\tau+r\rangle Z^{\beta} \square u(\tau, \cdot)\right\|_{2}\right],
\end{aligned}
$$

using (5.4), (5.6), (5.8), and (5.9) in the last step. If $|\beta| \leq 49$

$$
\langle\tau+r\rangle\left|Z^{\beta} \square u(\tau, y)\right| \leq \sum_{|\gamma| \leq 50}\left|Z^{\gamma} u^{\prime}(\tau, y)\right| \times\left(\langle\tau+r\rangle \sum_{|\gamma| \leq 25}\left|Z^{\gamma} u^{\prime}(\tau, y)\right|\right),
$$

which by the low energy estimate (5.8) and the low dispersive estimate (5.9) gives

$$
\left\|\langle\tau+r\rangle Z^{\beta} \square u(\tau, \cdot)\right\|_{2} \leq C \varepsilon \sup _{y}\langle\tau+r\rangle \sum_{|\gamma| \leq 25}\left|Z^{\gamma} u^{\prime}(\tau, y)\right| \leq C \varepsilon^{2} .
$$


Combining this with (5.28) and recalling that $(1-\eta(\tau, y))=0$ for $|y|>10 \tau$, we get

$$
I I I \leq C \varepsilon^{2} \sup _{0 \leq s \leq t}(1+s) \int_{s / 100}^{s} \int_{|r-(s-\tau)|<10}\left(\frac{1}{r^{1 / 2} \tau}\right)^{2} r d r d \tau \leq C \varepsilon^{2}
$$

which completes the proof of (ii.).

To complete the proof of Theorem 1.1 we need to show how (5.8), (15.9) imply (5.10)(5.13).

Since (5.9) has been established, the remainder of the argument follows nearly verbatim from the arguments of 22 . For completeness, we will sketch the argument here. We begin by using the above facts to prove (5.11). With notation as in $\S 1-2, \square_{\gamma} u=B(d u)$ with

$$
\gamma^{I J, j k}=-\sum_{\substack{0 \leq l \leq 3 \\ 1 \leq K \leq D}} B_{K . l}^{I J, j k} \partial_{l} u^{K}
$$

By (5.9), we have

$$
\left\|\gamma^{\prime}(s, \cdot)\right\|_{\infty} \leq \frac{C \varepsilon}{1+s}
$$

Let us first show the estimates for the energy of $\partial_{t}^{j} u$ for $j \leq M \leq 100$. We shall use induction on $M$.

We first notice that by (2.5) and (5.9) we have

$$
\partial_{t} E_{M}^{1 / 2}(u)(t) \leq C \sum_{j \leq M}\left\|\square_{\gamma} \partial_{t}^{j} u(t, \cdot)\right\|_{2}+\frac{C \varepsilon}{1+t} E_{M}^{1 / 2}(u)(t) .
$$

Note that for $M=1,2, \ldots$

$$
\begin{aligned}
\sum_{j \leq M}\left|\square_{\gamma} \partial_{t}^{j} u\right| \leq C\left(\sum_{j \leq M}\left|\partial_{t}^{j} u^{\prime}\right|\right. & \left.+\sum_{j \leq M-1}\left|\partial_{t}^{j} \partial^{2} u\right|\right) \sum_{|\alpha| \leq 40}\left|\partial^{\alpha} u^{\prime}\right| \\
& +C \sum_{|\alpha| \leq M-40}\left|\partial^{\alpha} u^{\prime}\right| \sum_{41 \leq|\alpha| \leq M / 2}\left|\partial^{\alpha} u^{\prime}\right| \\
& \leq \frac{C \varepsilon}{1+t}\left(\sum_{j \leq M}\left|\partial_{t}^{j} u^{\prime}\right|+\sum_{j \leq M-1}\left|\partial_{t}^{j} \partial^{2} u\right|\right)+C \sum_{|\alpha| \leq M-40}\left|\partial^{\alpha} u^{\prime}\right| \sum_{41 \leq|\alpha| \leq M / 2}\left|\partial^{\alpha} u^{\prime}\right|
\end{aligned}
$$

by (5.9) and (5.4). Also, if we use elliptic regularity and repeat this argument, we get

$$
\begin{gathered}
\sum_{j \leq M-1}\left\|\partial_{t}^{j} \partial^{2} u(t, \cdot)\right\|_{2} \leq C \sum_{j \leq M}\left\|\partial_{t}^{j} u^{\prime}(t, \cdot)\right\|_{2}+C \sum_{j \leq M-1}\left\|\partial_{t}^{j} \square u(t, \cdot)\right\|_{2} \\
\leq C \sum_{j \leq M}\left\|\partial_{t}^{j} u^{\prime}(t, \cdot)\right\|_{2}+\frac{C \varepsilon}{1+t} \sum_{j \leq M-1}\left\|\partial_{t}^{j} \partial^{2} u(t, \cdot)\right\|_{2} \\
+C \sum_{|\alpha| \leq M-41,|\beta| \leq M / 2}\left\|\partial^{\alpha} u^{\prime}(t, \cdot) \partial^{\beta} u^{\prime}(t, \cdot)\right\|_{2} \cdot
\end{gathered}
$$


If $\varepsilon$ is small, we can absorb the second to last term into the left side of the preceding inequality. Therefore, if we combine the last two inequalities, we conclude that

$$
\begin{aligned}
\sum_{j \leq M}\left\|\square_{\gamma} \partial_{t}^{j} u(t, \cdot)\right\|_{2} \leq \frac{C \varepsilon}{1+t} \sum_{j \leq M}\left\|\partial_{t}^{j} u^{\prime}(t, \cdot)\right\|_{2} & \\
& +C \sum_{|\alpha| \leq M-40,|\beta| \leq M / 2}\left\|\partial^{\alpha} u^{\prime}(t, \cdot) \partial^{\beta} u^{\prime}(t, \cdot)\right\|_{2} .
\end{aligned}
$$

If we combine this with (5.30) we get that for small $\varepsilon>0$

$$
\partial_{t} E_{M}^{1 / 2}(u)(t) \leq \frac{C \varepsilon}{1+t} E_{M}^{1 / 2}(u)(t)+C \sum_{|\alpha| \leq M-40,|\beta| \leq M / 2}\left\|\partial^{\alpha} u^{\prime}(t, \cdot) \partial^{\beta} u^{\prime}(t, \cdot)\right\|_{2},
$$

since when $\varepsilon$ is small, $\frac{1}{2} E_{M}^{1 / 2}(u)(t) \leq \sum_{j \leq M}\left\|\partial_{t}^{j} u^{\prime}(t, \cdot)\right\|_{2} \leq 2 E_{M}^{1 / 2}(u)(t)$.

For $M \leq 52$, the energy estimate (5.11) follows from (5.8). When $M>52$ we have to deal with the last term in (5.31). To do this we first note that by Lemma 4.4 we have

$$
\sum_{|\alpha| \leq M-40,|\beta| \leq M / 2}\left\|\partial^{\alpha} u^{\prime}(t, \cdot) \partial^{\beta} u^{\prime}(t, \cdot)\right\|_{2} \leq C \sum_{|\gamma| \leq \max (M-38,2+M / 2)}\left\|\langle x\rangle^{-1 / 2} Z^{\gamma} u^{\prime}(t, \cdot)\right\|_{2}^{2},
$$

which means that for $40 \leq M \leq 100$, (5.31), (5.1), and Gronwall's inequality yield

$$
E_{M}^{1 / 2}(u)(t) \leq C(1+t)^{C \varepsilon}\left[\varepsilon+\sum_{|\alpha| \leq \max (M-38,2+M / 2)}\left\|\langle x\rangle^{-1 / 2} Z^{\alpha} u^{\prime}\right\|_{L^{2}\left(S_{t}\right)}^{2}\right]
$$

if, as before, $S_{t}=[0, t] \times \mathbb{R}^{3} \backslash \mathcal{K}$.

If we use (5.8) and (5.32) along with a simple induction argument we conclude that we would have the desired bounds

$$
E_{100}^{1 / 2}(u)(t) \leq C \varepsilon(1+t)^{C \varepsilon+\sigma}
$$

for arbitrarily small $\sigma>0$ if we apply the following lemma.

Lemma 5.2. Under the above assumptions, if $M \leq 100-8 \mu, \mu \leq 4$, and

$$
\begin{aligned}
& \sum_{\substack{|\alpha|+\nu \leq M \\
\nu \leq \mu}}\left\|L^{\nu} \partial^{\alpha} u^{\prime}(t, \cdot)\right\|_{2}+\sum_{\substack{|\alpha|+\nu \leq M-3 \\
\nu \leq \mu}}\left\|\langle x\rangle^{-1 / 2} L^{\nu} \partial^{\alpha} u^{\prime}\right\|_{L^{2}\left(S_{t}\right)} \\
& +\sum_{\substack{|\alpha|+\nu \leq M-4 \\
\nu \leq \mu}}\left\|L^{\nu} Z^{\alpha} u^{\prime}(t, \cdot)\right\|_{2}+\sum_{\substack{|\alpha|+\nu \leq M-6 \\
\nu \leq \mu}}\left\|\langle x\rangle^{-1 / 2} L^{\nu} Z^{\alpha} u^{\prime}\right\|_{L^{2}\left(S_{t}\right)} \leq C \varepsilon(1+t)^{C \varepsilon+\sigma}
\end{aligned}
$$

with $\sigma>0$, then there is a constant $C^{\prime}$ so that

$$
\begin{gathered}
\sum_{\substack{|\alpha|+\nu \leq M-2 \\
\nu \leq \mu}}\left\|\langle x\rangle^{-1 / 2} L^{\nu} \partial^{\alpha} u^{\prime}\right\|_{L^{2}\left(S_{t}\right)}+\sum_{\substack{|\alpha|+\nu \leq M-3 \\
\nu \leq \mu}}\left\|L^{\nu} Z^{\alpha} u^{\prime}(t, \cdot)\right\|_{2} \\
+\sum_{\substack{|\alpha|+\nu \leq M-5 \\
\nu \leq \mu}}\left\|\langle x\rangle^{-1 / 2} L^{\nu} Z^{\alpha} u^{\prime}\right\|_{L^{2}\left(S_{t}\right)} \leq C^{\prime} \varepsilon(1+t)^{C^{\prime} \varepsilon+C^{\prime} \sigma} .
\end{gathered}
$$


The proof of this lemma can be found in 22 .

By elliptic regularity and (5.33), we get (5.11). Also, from Lemma 5.2 we get

$$
\begin{aligned}
\sum_{|\alpha| \leq 98}\left\|\langle x\rangle^{-1 / 2} \partial^{\alpha} w^{\prime}\right\|_{L^{2}\left(S_{t}\right)} & +\sum_{|\alpha| \leq 97}\left\|Z^{\alpha} w^{\prime}(t, \cdot)\right\|_{2} \\
& +\sum_{|\alpha| \leq 95}\left\|\langle x\rangle^{-1 / 2} Z^{\alpha} w^{\prime}\right\|_{L^{2}\left(S_{t}\right)} \leq C^{\prime} \varepsilon(1+t)^{C^{\prime} \varepsilon+C^{\prime} \sigma},
\end{aligned}
$$

since the same sort of bounds hold when $w$ is replaced by $u$.

Here and in what follows $\sigma$ denotes a small constant that must be taken to be larger and larger at each occurrence. Note that in terms of the number of $Z$ derivatives (5.35) is considerably stronger than the variants of (5.12) and (5.13) where one just takes the terms with $\nu=0$. This is because just as there is a loss of derivatives in going from (5.11) to (5.36), there will also be a loss of derivatives in going from $L^{2}$ bounds for terms of the form $L^{\nu} Z^{\alpha} u^{\prime}$ to those of the form $L^{\nu+1} Z^{\alpha} u^{\prime}$.

The proof of the estimates involving powers of $L$ is a bit more complicated, but still follows the strategy above. First we will estimate $L^{\nu} \partial^{\alpha} u^{\prime}$ in $L^{2}$ when $\alpha$ is small using (5.9). Then we shall estimate the remaining parts of (5.12) and (5.13) using Lemma 5.2

The main part of the next step is to show that

$$
\sum_{\substack{|\alpha|+\mu \leq 92 \\ \mu \leq 1}}\left\|L^{\mu} \partial^{\alpha} u^{\prime}(t, \cdot)\right\|_{2} \leq C \varepsilon(1+t)^{C \varepsilon+\sigma} .
$$

For this we shall want to use (2.8). We must first establish appropriate versions of (2.7) for $N_{0}+\nu_{0} \leq 92, \nu_{0}=1$. For this we note that for $M \leq 92$

$$
\begin{aligned}
& \sum_{\substack{j+\mu \leq M \\
\mu \leq 1}}\left(\left|\tilde{L}^{\mu} \partial_{t}^{j} \square_{\gamma} u\right|+\left|\left[\tilde{L}^{\mu} \partial_{t}^{j}, \square-\square_{\gamma}\right] u\right|\right) \\
& \leq C\left(\sum_{j \leq M-1}\left|\tilde{L} \partial_{t}^{j} \partial u\right|+\sum_{j \leq M-2}\left|\tilde{L} \partial_{t}^{j} \partial^{2} u\right|\right) \sum_{|\alpha| \leq 40}\left|\partial^{\alpha} u^{\prime}\right| \\
& +C \sum_{|\alpha| \leq M-41}\left|L \partial^{\alpha} u^{\prime}\right| \sum_{|\alpha| \leq M}\left|\partial^{\alpha} u^{\prime}\right|+C \sum_{|\alpha| \leq M}\left|\partial^{\alpha} u^{\prime}\right| \sum_{|\alpha| \leq \max (M / 2, M-40)}\left|\partial^{\alpha} u^{\prime}\right| .
\end{aligned}
$$

By this, (5.9), and elliptic regularity, we get that for $M \leq 92$

$$
\begin{gathered}
\sum_{\substack{j+\mu \leq M \\
\mu \leq 1}}\left(\left\|\tilde{L}^{\mu} \partial_{t}^{j} \square_{\gamma} u(t, \cdot)\right\|_{2}+\left\|\left[\tilde{L}^{\mu} \partial_{t}^{j}, \square-\square_{\gamma}\right] u(t, \cdot)\right\|_{2}\right) \leq \frac{C \varepsilon}{1+t} \sum_{j+\mu \leq M}\left\|\tilde{L}_{\mu \leq 1}^{\mu} \partial_{t}^{j} u^{\prime}(t, \cdot)\right\|_{2} \\
+C \sum_{|\alpha| \leq M-41}\left\|\langle x\rangle^{-1 / 2} L \partial^{\alpha} u^{\prime}(t, \cdot)\right\|_{2} \sum_{|\alpha| \leq 94}\left\|\langle x\rangle^{-1 / 2} Z^{\alpha} u^{\prime}(t, \cdot)\right\|_{2} \\
+C \sum_{|\alpha| \leq \max (M, 2+M / 2)}\left\|\langle x\rangle^{-1 / 2} Z^{\alpha} u^{\prime}(t, \cdot)\right\|_{2}^{2} .
\end{gathered}
$$


Based on this if $\varepsilon$ is small then (2.7) holds with $\delta=C \varepsilon$ and

$$
H_{1, M-1}(t)=C \sum_{|\alpha| \leq M-41}\left\|\langle x\rangle^{-1 / 2} L \partial^{\alpha} u^{\prime}(t, \cdot)\right\|_{2}^{2}+C \sum_{|\alpha| \leq 94}\left\|\langle x\rangle^{-1 / 2} Z^{\alpha} u^{\prime}(t, \cdot)\right\|_{2}^{2} .
$$

Therefore since the conditions on the data give $\int e_{0}\left(\tilde{L}^{\nu} \partial_{t}^{j} u\right)(0, x) d x \leq C \varepsilon^{2}$ if $\nu+j \leq 100$ it follows from (2.8) and (5.36) that for $M \leq 92$

$$
\begin{gathered}
\sum_{\substack{|\alpha|+\mu \leq M \\
\mu \leq 1}}\left\|L^{\mu} \partial^{\alpha} u^{\prime}(t, \cdot)\right\|_{2} \leq C \varepsilon(1+t)^{C \varepsilon+\sigma}+C(1+t)^{C \varepsilon} \sum_{|\alpha| \leq M-41}\left\|\langle x\rangle^{-1 / 2} L \partial^{\alpha} u^{\prime}\right\|_{L^{2}\left(S_{t}\right)}^{2} \\
+C(1+t)^{C \varepsilon} \int_{0}^{t} \sum_{|\alpha| \leq M+1}\left\|\partial^{\alpha} u^{\prime}(s, \cdot)\right\|_{L^{2}(|x|<1)} d s .
\end{gathered}
$$

If we apply (2.13) and (5.4) we get that the last integral is dominated by $\varepsilon \log (2+t)$ plus

$$
\begin{aligned}
& \int_{0}^{t} \sum_{|\alpha| \leq M+1}\left\|\partial^{\alpha} w^{\prime}(s, \cdot)\right\|_{L^{2}(|x|<1)} d s \\
& \leq C \sum_{|\alpha| \leq M+2} \int_{0}^{t}\left(\int_{0}^{s}\left\|\partial^{\alpha} \square w(\tau, \cdot)\right\|_{L^{2}(|| x|-(s-\tau)|<10)} d \tau\right) d s .
\end{aligned}
$$

By (5.4) if we replace $w$ by $u_{0}$ then the analog of the last term is $O(\varepsilon \log (2+t))$. We therefore conclude that

$$
\begin{aligned}
& \sum_{|\alpha| \leq M+1} \int_{0}^{t}\left\|\partial^{\alpha} u^{\prime}(s, \cdot)\right\|_{L^{2}(|x|<1)} d s \leq C \varepsilon \log (2+t) \\
& +C \sum_{|\alpha| \leq M+2} \int_{0}^{t}\left(\int_{0}^{s}\left\|\partial^{\alpha} \square u(\tau, \cdot)\right\|_{L^{2}(|| x|-(s-\tau)|<10)} d \tau\right) d s .
\end{aligned}
$$

Since

$$
\sum_{|\alpha| \leq M+2}\left|\partial^{\alpha} \square u\right| \leq C \sum_{|\alpha| \leq M+3}\left|\partial^{\alpha} u^{\prime}\right| \sum_{|\alpha| \leq(M+3) / 2}\left|\partial^{\alpha} u^{\prime}\right|
$$

an application of Lemma 4.4 yields

$$
\sum_{|\alpha| \leq M+2}\left\|\partial^{\alpha} \square u(\tau, \cdot)\right\|_{L^{2}(|| x|-(s-\tau)|<10)} \leq C \sum_{|\alpha| \leq 95}\left\|\langle x\rangle^{-1 / 2} Z^{\alpha} u^{\prime}\right\|_{L^{2}(|| x|-(s-\tau)|<20)}^{2},
$$

since $(3+M) / 2 \leq 95$ if $M \leq 92$. Since the sets $\{(\tau, x):|| x|-(j-\tau)|<20\}, j=0,1,2, \ldots$ have finite overlap, we conclude that for $M \leq 92$

$$
\begin{aligned}
\sum_{|\alpha| \leq M+1} \int_{0}^{t}\left\|\partial^{\alpha} u^{\prime}(s, \cdot)\right\|_{L^{2}(|x|<1)} d s & \leq C \varepsilon \log (2+t)+C \sum_{|\alpha| \leq 95}\left\|\langle x\rangle^{-1 / 2} Z^{\alpha} u^{\prime}\right\|_{L^{2}\left(S_{t}\right)}^{2} \\
& \leq C \varepsilon(1+t)^{C \varepsilon+\sigma} .
\end{aligned}
$$

Therefore, by (5.38) we have that

$$
\sum_{\substack{|\alpha|+\mu \leq M \\ \mu \leq 1}}\left\|L^{\mu} \partial^{\alpha} u^{\prime}(t, \cdot)\right\|_{2} \leq C \varepsilon(1+t)^{C \varepsilon+\sigma}+C(1+t)^{C \varepsilon} \sum_{|\alpha| \leq M-41}\left\|\langle x\rangle^{-1 / 2} L \partial^{\alpha} u^{\prime}\right\|_{L^{2}\left(S_{t}\right)}^{2} .
$$


This gives the desired bounds when $M \leq 40$.

If we now use induction and Lemma 5.2 we get (5.37) as well as

$$
\begin{gathered}
\sum_{\substack{|\alpha|+\mu \leq 90 \\
\mu \leq 1}}\left\|\langle x\rangle^{-1 / 2} L^{\mu} \partial^{\alpha} u^{\prime}\right\|_{L^{2}\left(S_{t}\right)}+\sum_{\substack{|\alpha|+\mu \leq 89 \\
\mu \leq 1}}\left\|L^{\mu} Z^{\alpha} u^{\prime}(t, \cdot)\right\|_{2} \\
+\sum_{\substack{|\alpha|+\mu \leq 87 \\
\mu \leq 1}}\left\|\langle x\rangle^{-1 / 2} L^{\mu} Z^{\alpha} u^{\prime}\right\|_{L^{2}\left(S_{t}\right)} \leq C \varepsilon(1+t)^{C \varepsilon+C \sigma} .
\end{gathered}
$$

If we repeat this argument we can estimate $L^{2} Z^{\alpha} u^{\prime}, L^{3} Z^{\alpha} u^{\prime}$, and $L^{4} Z^{\alpha} u^{\prime}$ for appropriate $Z^{\alpha}$. Using (5.37), (5.39), and the last argument gives

$$
\begin{gathered}
\sum_{\substack{|\alpha|+\mu \leq 84 \\
\mu \leq 2}}\left\|L^{\mu} \partial^{\alpha} u^{\prime}(t, \cdot)\right\|_{2}+\sum_{\substack{|\alpha|+\mu \leq 82 \\
\mu \leq 2}}\left\|\langle x\rangle^{-1 / 2} L^{\mu} \partial^{\alpha} u^{\prime}\right\|_{L^{2}\left(S_{t}\right)}+\sum_{\substack{|\alpha|+\mu \leq 81 \\
\mu \leq 2}}\left\|L^{\mu} Z^{\alpha} u^{\prime}(t, \cdot)\right\|_{2} \\
+\sum_{\substack{|\alpha|+\mu \leq 79 \\
\mu \leq 2}}\left\|\langle x\rangle^{-1 / 2} L^{\mu} Z^{\alpha} u^{\prime}\right\|_{L^{2}\left(S_{t}\right)} \leq C \varepsilon(1+t)^{C \varepsilon+C \sigma} .
\end{gathered}
$$

Then using the estimates for $L^{\mu} Z^{\alpha} u^{\prime}, \mu \leq 2$ we can argue as above to get

$$
\begin{gathered}
\sum_{\substack{|\alpha|+\mu \leq 76 \\
\mu \leq 3}}\left\|L^{\mu} \partial^{\alpha} u^{\prime}(t, \cdot)\right\|_{2}+\sum_{\substack{|\alpha|+\mu \leq 74 \\
\mu \leq 2}}\left\|\langle x\rangle^{-1 / 2} L^{\mu} \partial^{\alpha} u^{\prime}\right\|_{L^{2}\left(S_{t}\right)}+\sum_{\substack{|\alpha|+\mu \leq 73 \\
\mu \leq 3}}\left\|L^{\mu} Z^{\alpha} u^{\prime}(t, \cdot)\right\|_{2} \\
+\sum_{\substack{|\alpha|+\mu \leq 71 \\
\mu \leq 3}}\left\|\langle x\rangle^{-1 / 2} L^{\mu} Z^{\alpha} u^{\prime}\right\|_{L^{2}\left(S_{t}\right)} \leq C \varepsilon(1+t)^{C \varepsilon+C \sigma} .
\end{gathered}
$$

Similarly, using the estimates for $L^{\mu} Z^{\alpha} u^{\prime}$ for $\mu \leq 3$ we finally get

$$
\begin{gathered}
\sum_{\substack{|\alpha|+\mu \leq 68 \\
\mu \leq 4}}\left\|L^{\mu} \partial^{\alpha} u^{\prime}(t, \cdot)\right\|_{2}+\sum_{\substack{|\alpha|+\mu \leq 66 \\
\mu \leq 2}}\left\|\langle x\rangle^{-1 / 2} L^{\mu} \partial^{\alpha} u^{\prime}\right\|_{L^{2}\left(S_{t}\right)}+\sum_{\substack{|\alpha|+\mu \leq 65 \\
\mu \leq 4}}\left\|L^{\mu} Z^{\alpha} u^{\prime}(t, \cdot)\right\|_{2} \\
+\sum_{\substack{|\alpha|+\mu \leq 63 \\
\mu \leq 4}}\left\|\langle x\rangle^{-1 / 2} L^{\mu} Z^{\alpha} u^{\prime}\right\|_{L^{2}\left(S_{t}\right)} \leq C \varepsilon(1+t)^{C \varepsilon+C \sigma} .
\end{gathered}
$$

If we combine this with our earlier bounds, we conclude that (15.12) and (15.13) must be valid.

It remains to prove (5.10). This is straightforward. If we use Theorem 3.1 we find that its left side is dominated by the square of that of (5.13). Hence (5.13) implies (5.10) which completes the proof.

\section{REFERENCES}

[1] R. Agemi and K. Yokoyama: The null condition and global existence of solutions to systems of wave equations with different speeds, Advances in Nonlinear Partial Differential Equations and Stochastics, (1998), 43-86.

[2] N. Burq: Décroissance de lénergie locale de l'équation des ondes pour le problìe extérieur et absence de rśonance au voisinage du réel, Acta Math. 180 (1998), 1-29. 
[3] D. Christodoulou: Global solutions of nonlinear hyperbolic equations for small initial data, Comm. Pure Appl. Math. 39 (1986), 267-282.

[4] D. Gilbarg and N. Trudinger: Elliptic partial differential equations of second order, Springer, Second Ed., Third Printing, 1998.

[5] K. Hidano: An elementary proof of global or almost global existence for quasi-linear wave equations, Tohoku Math. J. (2), 56 (2004), 271-287.

[6] K. Hidano and K. Yokoyama: A remark on the almost global existence theorems of Keel, Smith, and Sogge, preprint.

[7] L. Hörmander: Lectures on nonlinear hyperbolic equations, Springer-Verlag, Berlin, 1997.

[8] L. Hörmander: $L^{1}, L^{\infty}$ estimates for the wave operator, Analyse Mathematique et Applications, Gauthier-Villars, Paris, 1988, pp. 211-234.

[9] M. Ikawa: Decay of solutions of the wave equation in the exterior of two convex bodies, Osaka J. Math. 19 (1982), 459-509.

[10] M. Ikawa: Decay of solutions of the wave equation in the exterior of several convex bodies, Ann. Inst. Fourier (Grenoble), 38 (1988), 113-146.

[11] F. John, Nonlinear wave equations, formation of singularities, Amer. Math. Soc., 1990.

[12] M. Keel, H. Smith, and C. D. Sogge: Global existence for a quasilinear wave equation outside of star-shaped domains, J. Funct. Anal. 189 (2002), 155-226.

[13] M. Keel, H. Smith, and C. D. Sogge: Almost global existence for some semilinear wave equations, J. D'Analyse 87 (2002), 265-279.

[14] M. Keel, H. Smith, and C. D. Sogge: Almost global existence for quasilinear wave equations in three space dimensions, J. Amer. Math. Soc. 17 (2004), 109-153.

[15] S. Klainerman: Uniform decay estimates and the Lorentz invariance of the classical wave equation, Comm. Pure Appl. Math. 38 (1985), 321-332.

[16] S. Klainerman: The null condition and global existence to nonlinear wave equations, Lectures in Applied Math. 23 (1986), 293-326.

[17] S. Klainerman and T. Sideris: On almost global existence for nonrelativistic wave equations in $3 d$ Comm. Pure Appl. Math. 49 (1996), 307-321.

[18] K. Kubota and K. Yokoyama: Global existence of classical solutions to systems of nonlinear wave equations with different speeds of propagation, Japan. J. Math. 27 (2001), 113-202.

[19] P. D. Lax, C. S. Morawetz, and R. S. Phillips: Exponential decay of solutions of the wave equation in the exterior of a star-shaped obstacle, Comm. Pure Appl. Math. 16 (1963), 477-486.

[20] P. D. Lax and R. S. Phillips: Scattering theory, revised edition, Academic Press, San Diego, 1989.

[21] R. B. Melrose: Singularities and energy decay of acoustical scattering, Duke Math. J. 46 (1979), $43-59$.

[22] J. Metcalfe and C. D. Sogge: Hyperbolic trapped rays and global existence of quasilinear wave equations, Invent. Math., to appear.

[23] C. S. Morawetz: The decay of solutions of the exterior initial-boundary problem for the wave equation, Comm. Pure Appl. Math. 14 (1961), 561-568.

[24] C. S. Morawetz, J. Ralston, and W. Strauss: Decay of solutions of the wave equation outside nontrapping obstacles, Comm. Pure Appl. Math. 30 (1977), 447-508.

[25] J. V. Ralston: Solutions of the wave equation with localized energy, Comm. Pure Appl. Math. 22 (1969), 807-923.

[26] Y. Shibata and Y. Tsutsumi: On a global existence theorem of small amplitude solutions for nonlinear wave equations in an exterior domain, Math. Z. 191 (1986), 165-199.

[27] T. Sideris: Nonresonance and global existence of prestressed nonlinear elastic waves, Ann. of Math. 151 (2000), 849-874.

[28] T. Sideris: The null condition and global existence of nonlinear elastic waves., Inven. Math. 123 (1996), 323-342.

[29] T. Sideris, S.Y. Tu: Global existence for systems of nonlinear wave equations in 3D with multiple speeds, SIAM J. Math. Anal. 33 (2001), 477-488.

[30] H. Smith and C. D. Sogge: Global Strichartz estimates for nontrapping perturbations of the Laplacian, Comm. Partial Differential Equations 25 (2000), 2171-2183.

[31] C. D. Sogge: Lectures on nonlinear wave equations, International Press, Cambridge, MA, 1995. 
[32] C.D. Sogge: Global existence for nonlinear wave equations with multiple speeds, Harmonic analysis at Mount Holyoke (South Hadley, MA, 2001), 353-366, Contemp. Math., 320, Amer. Math. Soc., Providence, RI, 2003.

[33] K. Yokoyama: Global existence of classical solutions to systems of wave equations with critical nonlinearity in three space dimensions J. Math. Soc. Japan 52 (2000), 609-632.

School of Mathematics, Georgia Institute of Technology, Atlanta, GA 30332-0160

Graduate School of Information Sciences, Tohoku University, Sendai 980-8579, Japan

Department of Mathematics, Johns Hopkins University, Baltimore, MD 21218 